July 23,2018

BA-TH/99-359

\title{
Spontaneous emission and lifetime modification caused by an intense electromagnetic field
}

\author{
P. FACCHI and S. PASCAZIO \\ Dipartimento di Fisica, Università di Bari \\ and Istituto Nazionale di Fisica Nucleare, Sezione di Bari \\ I-70126 Bari, Italy
}

PACS: 42.50.Hz; 42.50.Vk; 03.65.Bz

\begin{abstract}
We study the temporal evolution of a three-level system (such as an atom or a molecule), initially prepared in an excited state, bathed in a laser field tuned at the transition frequency of the other level. The features of the spontaneous emission are investigated and the lifetime of the initial state is evaluated: a Fermi "golden rule" still applies, but the on-shell matrix elements depend on the intensity of the laser field. In general, the lifetime is a decreasing function of the laser intensity. The phenomenon we discuss can be viewed as an "inverse" quantum Zeno effect and can be analyzed in terms of dressed states.
\end{abstract}




\section{Introduction}

The temporal behavior of quantum mechanical systems can be strongly influenced by the action of an external agent. A good example is the quantum Zeno effect [1], 2], where the quantum mechanical evolution of a given (not necessarily unstable) state is slowed down (or even halted) by performing a series of measurements that ascertain whether the system is still in its initial state. This peculiar effect is historically associated and usually ascribed to what we could call a "pulsed" quantum mechanical observation on the system. However, it can also be obtained by performing a "continuous" observation of the quantum state, e.g. by means of an intense field [3], 4 .

Most experiment that have been performed or proposed in order to modify the quantum mechanical evolution law make use of oscillating systems [5, 6, 7, 8, 9]. On the other hand, it would be interesting to understand whether and to which extent the evolution law of a bona fide "unstable" system can be changed. In order to discuss the evolution of genuine unstable systems one usually makes use of the WeisskopfWigner approximation [10], that ascribes the main properties of the decay law to a pole located near the real axis of the complex energy plane. This yields the Fermi "golden rule" [11]. In this paper we shall investigate the possibility that the lifetime of an unstable quantum system can be modified by the presence of a very intense electromagnetic field. We shall look at the temporal behavior of a three-level system (such as an atom or a molecule), where level \#1 is the ground state and levels \#2, \#3 are two excited states. (See Figure 11.) The system is initially prepared in level $\# 2$ and if it follows its natural evolution, it will decay to level \#1. The decay will be (approximately) exponential and characterized by a certain lifetime, that can be calculated from the Fermi golden rule. But if one shines on the system an intense laser field, tuned at the transition frequency $3-1$, the evolution can be different. This problem was investigated in Ref. [3], where it was found that the lifetime of the initial state depends on the intensity of the laser field. In the limit of an extremely intense field, the initial state undergoes a "continuous observation" and the decay should be considerably slowed down (quantum Zeno effect). The aim of this paper is to study this effect in more detail and discuss a new phenomenon [12]: we shall see that for physically sensible values of the intensity of the laser, the decay can be enhanced, rather than hindered. This can be viewed as an "inverse" quantum Zeno effect. An important role in this context will be played by the specific properties of the interaction Hamiltonian, in particular by the "form factor" of the interaction.

Other authors have studied physical effects that are related to those we shall discuss. The features of the matrix elements of the interaction Hamiltonian were investigated in the context of the quantum Zeno effect by Kofman and Kurizki [13], who also emphasized that different quantum Zeno regimes are present. Plenio, Knight and Thompson discussed the quantum Zeno effect due to "continuous" measurements and considered several physical systems whose evolution is modified by an external field [14]. There is also work by Kraus on a similar subject [15]. Finally, Zhu, Narducci and Scully [16] investigated the electromagnetic-induced transparency in a context 
similar to that considered in this paper. In some sense, our present investigation "blends" these studies, by taking into account the important role played by the matrix elements of the interaction. This will enable us to discuss some new features of the evolution that have not been considered before. We shall look at this phenomenon from several perspectives, by first solving the time-dependent Schrödinger equation, then looking at the spectrum of the emitted photons and finally constructing the dressed (Fano) states.

Our analysis will be performed within the Weisskopf-Wigner approximation and no deviations at short [1, 17, 18] and long [19] times will be considered. The features of the quantum mechanical evolution are summarized in [20 and have already been discussed within a quantum field theoretical framework [21, 22, 23, 24, where several subtle effects have to be properly taken into account.

This paper is organized as follows: in Section 2 we introduce the 3-level system bathed in the laser field. Its temporal evolution is studied in Section 3. The spectrum of the photons emitted during the evolution is evaluated in Section 4. Section 5 contains a discussion in terms of dressed states, Section 6 an analysis of the influence of additional levels on the lifetime and Section 7 some concluding remarks.

\section{Preliminaries and definitions}

We consider the Hamiltonian $(\hbar=c=1)[3]$ :

$$
\begin{aligned}
H= & H_{0}+H_{\mathrm{int}} \\
= & \omega_{0}|2\rangle\left\langle 2\left|+\Omega_{0}\right| 3\right\rangle\langle 3|+\sum_{\boldsymbol{k}, \lambda} \omega_{k} a_{\boldsymbol{k} \lambda}^{\dagger} a_{\boldsymbol{k} \lambda}+\sum_{\boldsymbol{k}, \lambda}\left(\phi_{\boldsymbol{k} \lambda} a_{\boldsymbol{k} \lambda}^{\dagger}|1\rangle\left\langle 2\left|+\phi_{\boldsymbol{k} \lambda}^{*} a_{\boldsymbol{k} \lambda}\right| 2\right\rangle\langle 1|\right) \\
& +\sum_{\boldsymbol{k}, \lambda}\left(\Phi_{\boldsymbol{k} \lambda} a_{\boldsymbol{k} \lambda}^{\dagger}|1\rangle\left\langle 3\left|+\Phi_{\boldsymbol{k} \lambda}^{*} a_{\boldsymbol{k} \lambda}\right| 3\right\rangle\langle 1|\right),
\end{aligned}
$$

where the first two terms are the free Hamiltonian of the 3-level atom (whose states $|i\rangle(i=1,2,3)$ have energies $\left.E_{1}=0, \omega_{0}=E_{2}-E_{1}>0, \Omega_{0}=E_{3}-E_{1}>0\right)$, the third term is the free Hamiltonian of the EM field and the last two terms describe the $1 \leftrightarrow 2$ and $1 \leftrightarrow 3$ transitions in the rotating wave approximation, respectively. (See Figure 1.) States $|2\rangle$ and $|3\rangle$ are chosen so that no transition between them is possible (e.g., because of selection rules). The matrix elements of the interaction Hamiltonian read

$$
\phi_{\boldsymbol{k} \lambda}=\frac{e}{\sqrt{2 \epsilon_{0} V \omega}} \int d^{3} x e^{-i \boldsymbol{k} \cdot \boldsymbol{x}} \boldsymbol{\epsilon}_{\boldsymbol{k} \lambda}^{*} \cdot \boldsymbol{j}_{12}(\boldsymbol{x}) \quad \Phi_{\boldsymbol{k} \lambda}=\frac{e}{\sqrt{2 \epsilon_{0} V \omega}} \int d^{3} x e^{-i \boldsymbol{k} \cdot \boldsymbol{x}} \boldsymbol{\epsilon}_{\boldsymbol{k} \lambda}^{*} \cdot \boldsymbol{j}_{13}(\boldsymbol{x}),
$$

where $-e$ is the electron charge, $\epsilon_{0}$ the vacuum permittivity, $V$ the volume of the box, $\omega=|\boldsymbol{k}|, \boldsymbol{\epsilon}_{\boldsymbol{k} \lambda}$ the photon polarization and $\boldsymbol{j}_{\mathrm{fi}}$ the transition current of the radiating system. For example, in the case of an electron in an external field, we have $\boldsymbol{j}_{\mathrm{fi}}=\psi_{\mathrm{f}}^{\dagger} \boldsymbol{\alpha} \psi_{\mathrm{i}}$ where $\psi_{\mathrm{i}}$ and $\psi_{\mathrm{f}}$ are the wavefunctions of the initial and final state, respectively, and $\boldsymbol{\alpha}$ is the vector of Dirac matrices. For the sake of generality we are using relativistic matrix elements, but our analysis can also be performed with nonrelativistic ones $\boldsymbol{j}_{\mathrm{fi}}=\psi_{\mathrm{f}}^{*} \boldsymbol{p} \psi_{\mathrm{i}} / m_{e}$, where $\boldsymbol{p} / m_{e}$ is the electron velocity. 


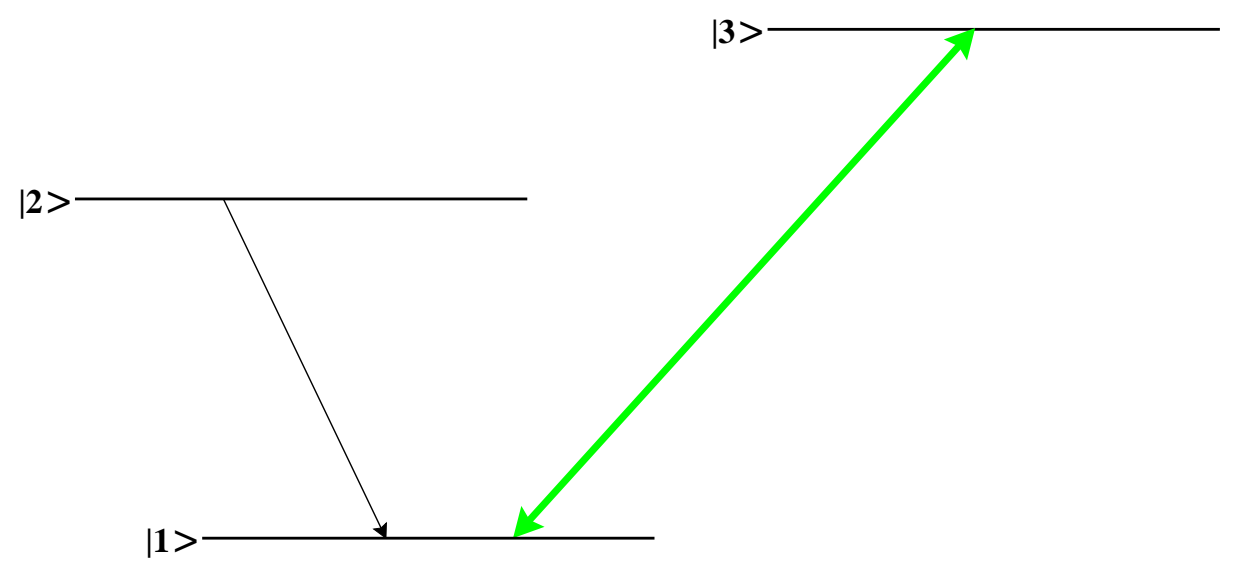

Figure 1: Level configuration

We shall concentrate our attention on a 3-level system bathed in a continuous laser beam, whose photons have momentum $\boldsymbol{k}_{0}\left(\left|\boldsymbol{k}_{0}\right|=\Omega_{0}\right)$ and polarization $\lambda_{0}$, and assume, throughout this paper, that

$$
\phi_{\boldsymbol{k}_{0} \lambda_{0}}=0
$$

i.e., the laser does not interact with state $|2\rangle$. Let the laser be in a coherent state $\left|\alpha_{0}\right\rangle$ with a very large average number $\bar{N}_{0}=\left|\alpha_{0}\right|^{2}$ of $\boldsymbol{k}_{0}$-photons in volume $V$ [we will eventually consider the thermodynamical limit; see Eq. (3.17)]. In the picture defined by the unitary operator

$$
T(t)=\exp \left(\alpha_{0}^{*} e^{i \Omega_{0} t} a_{\boldsymbol{k}_{0} \lambda_{0}}-\alpha_{0} e^{-i \Omega_{0} t} a_{\boldsymbol{k}_{0} \lambda_{0}}^{\dagger}\right),
$$

the Hamiltonian (2.1) reads

$$
H(t)=T H T^{\dagger}+i \dot{T} T^{\dagger}=H+\left(\Phi_{\boldsymbol{k}_{0} \lambda_{0}} \alpha_{0}^{*} e^{i \Omega_{0} t}|1\rangle\left\langle 3\left|+\Phi_{\boldsymbol{k}_{0} \lambda_{0}}^{*} \alpha_{0} e^{-i \Omega_{0} t}\right| 3\right\rangle\langle 1|\right) .
$$

In this picture, the $\boldsymbol{k}_{0}$ mode is initially in the vacuum state [25] and by noting that for $\bar{N}_{0} \gg 1$

$$
\left|\left\langle 1 ; 0_{\boldsymbol{k} \lambda}|H(t)| 3 ; 0_{\boldsymbol{k} \lambda}\right\rangle\right|=\sqrt{\bar{N}_{0}}\left|\Phi_{\boldsymbol{k}_{0} \lambda_{0}}\right| \gg\left|\left\langle 1 ; 1_{\boldsymbol{k} \lambda}|H(t)| 3 ; 0_{\boldsymbol{k} \lambda}\right\rangle\right|=\left|\Phi_{\boldsymbol{k} \lambda}\right|,
$$

the Hamiltonian (2.5) becomes

$$
\begin{aligned}
H \simeq & \omega_{0}|2\rangle\left\langle 2\left|+\Omega_{0}\right| 3\right\rangle\langle 3|+\sum_{\boldsymbol{k}, \lambda} \omega_{k} a_{\boldsymbol{k} \lambda}^{\dagger} a_{\boldsymbol{k} \lambda}+\sum_{\boldsymbol{k}, \lambda}^{\prime}\left(\phi_{\boldsymbol{k} \lambda} a_{\boldsymbol{k} \lambda}^{\dagger}|1\rangle\left\langle 2\left|+\phi_{\boldsymbol{k} \lambda}^{*} a_{\boldsymbol{k} \lambda}\right| 2\right\rangle\langle 1|\right) \\
& +\left(\Phi_{\boldsymbol{k}_{0} \lambda_{0}} \alpha_{0}^{*} e^{i \Omega_{0} t}|1\rangle\left\langle 3\left|+\Phi_{\boldsymbol{k}_{0} \lambda_{0}}^{*} \alpha_{0} e^{-i \Omega_{0} t}\right| 3\right\rangle\langle 1|\right)
\end{aligned}
$$

where a prime means that the summation does not include $\left(\boldsymbol{k}_{0}, \lambda_{0}\right)$ due to hypothesis $(2.3)]$. In the above equations and henceforth, the vector $\left|i ; n_{\boldsymbol{k} \lambda}\right\rangle$ represents a state in which the atom is in state $|i\rangle$ and the electromagnetic field in a state with $n_{\boldsymbol{k} \lambda}$ $(\boldsymbol{k}, \lambda)$-photons. We shall analyze the behavior of the system under the action of a 
continuous laser beam of high intensity. Under these conditions, level configurations similar to that of Figure 1 give rise to the phenomenon of induced transparency [26], for laser beams of sufficiently high intensities. Our interest, however, will be focused on unstable initial states: we shall study the temporal behavior of level \#2 when the system is shined by a continuous laser of intensity comparable to those used to obtain induced transparency.

Notice that in Eq. (2.7) the spontaneous decay $3 \rightarrow 1$ has been neglected with

respect to the stimulated transition, because of the large factor $\sqrt{\bar{N}_{0}} \gg 1$ in Eq. (2.6). However, since our interest is primarily in the first step of this process, namely the decay $2 \rightarrow 1$, these smaller, later effects (of the order of $1 / N_{0}$ ) do not change our conclusions.

The operator

$$
\mathcal{N}=|2\rangle\langle 2|+\sum_{\boldsymbol{k}, \lambda}^{\prime} a_{\boldsymbol{k} \lambda}^{\dagger} a_{\boldsymbol{k} \lambda}
$$

satisfies

$$
[H, \mathcal{N}]=0,
$$

which implies the conservation of the total number of photons plus the atomic excitation (Tamm-Dancoff approximation [27]). The Hilbert space splits therefore into sectors that are invariant under the action of the Hamiltonian: in our case, the system evolves in the subspace labelled by the eigenvalue $\mathcal{N}=1$ and the analysis can be restricted to this sector [28].

\section{Temporal evolution}

We will study the temporal evolution by solving the time-dependent Schrödinger equation

$$
i \frac{d}{d t}|\psi(t)\rangle=H(t)|\psi(t)\rangle
$$

where the states of the total system in the sector $\mathcal{N}=1$ read

$$
|\psi(t)\rangle=x(t)|2 ; 0\rangle+\sum_{\boldsymbol{k}, \lambda}^{\prime} y_{\boldsymbol{k} \lambda}(t)\left|1 ; 1_{\boldsymbol{k} \lambda}\right\rangle+\sum_{\boldsymbol{k}, \lambda}^{\prime} z_{\boldsymbol{k} \lambda}(t) e^{-i \Omega_{0} t}\left|3 ; 1_{\boldsymbol{k} \lambda}\right\rangle
$$

and are normalized:

$$
\langle\psi(t) \mid \psi(t)\rangle=|x(t)|^{2}+\sum_{\boldsymbol{k}, \lambda}^{\prime}\left|y_{\boldsymbol{k}, \lambda}(t)\right|^{2}+\sum_{\boldsymbol{k}, \lambda}^{\prime}\left|z_{\boldsymbol{k}, \lambda}(t)\right|^{2}=1
$$

By inserting (3.2) in (3.1) one obtains the equations of motion

$$
\begin{aligned}
i \dot{x}(t) & =\omega_{0} x(t)+\sum_{\boldsymbol{k}, \lambda}^{\prime} \phi_{\boldsymbol{k} \lambda}^{*} y_{\boldsymbol{k} \lambda}(t), \\
i \dot{y}_{\boldsymbol{k} \lambda}(t) & =\phi_{\boldsymbol{k} \lambda} x(t)+\omega_{k} y_{\boldsymbol{k} \lambda}(t)+\alpha_{0}^{*} \Phi_{\boldsymbol{k}_{0} \lambda_{0}} z_{\boldsymbol{k} \lambda}(t), \\
i \dot{z}_{\boldsymbol{k} \lambda}(t) & =\alpha_{0} \Phi_{\boldsymbol{k}_{0} \lambda_{0}}^{*} y_{\boldsymbol{k} \lambda}(t)+\omega_{k} z_{\boldsymbol{k} \lambda}(t),
\end{aligned}
$$


where a dot denotes time derivative. At time $t=0$ we prepare our system in the state

$$
|\psi(0)\rangle=|2 ; 0\rangle \quad \Leftrightarrow \quad x(0)=1, y_{\boldsymbol{k} \lambda}(0)=0, z_{\boldsymbol{k} \lambda}(0)=0,
$$

which is an eigenstate of the free Hamiltonian

$$
H_{0}|\psi(0)\rangle=H_{0}|2 ; 0\rangle=\omega_{0}|2 ; 0\rangle .
$$

Incidentally, we stress that the choice of the initial state is different from that of Ref. [5], where the 3-level atom is initially in the ground state (\#1) and a Rabi oscillation to level \#2, provoked by an rf-field, is inhibited by a pulsed laser, resonating between levels \#1 and \#3, that performs the "observation" of level \#1. In our case, the atom is initially in level \#2, so that it can spontaneously decay to level \#1, and it is "continuously observed" by a continuous laser at the 1-3 frequency [3]: As soon as the system has decayed to level \#1, the (intense) laser provokes the $1 \rightarrow 3$ transition. (The irreversibility inherent in the act of observation is eventually brought in by the spontaneous decay of level \#3.) This brings us conceptually closer to the seminal formulation [1], 2] of quantum Zeno effect.

By Laplace transforming the system of differential equations (3.4) and incorporating the initial condition (3.5) we get the algebraic system

$$
\begin{aligned}
i s \widetilde{x}(s) & =\omega_{0} \widetilde{x}(s)+\sum_{\boldsymbol{k}, \lambda}^{\prime} \phi_{\boldsymbol{k} \lambda}^{*} \widetilde{y}_{\boldsymbol{k} \lambda}(s)+i, \\
i s \widetilde{y}_{\boldsymbol{k} \lambda}(s) & =\phi_{\boldsymbol{k} \lambda} \widetilde{x}(s)+\omega_{k} \widetilde{y}_{\boldsymbol{k} \lambda}(s)+\alpha_{0}^{*} \Phi_{\boldsymbol{k}_{0} \lambda_{0}} \widetilde{z}_{\boldsymbol{k} \lambda}(s), \\
i s \widetilde{z}_{\boldsymbol{k} \lambda}(s) & =\alpha_{0} \Phi_{\boldsymbol{k}_{0} \lambda_{0}}^{*} \widetilde{y}_{\boldsymbol{k} \lambda}(s)+\omega_{k} \widetilde{z}_{\boldsymbol{k} \lambda}(s),
\end{aligned}
$$

where

$$
\widetilde{f}(s)=\int_{0}^{\infty} d t e^{-s t} f(t), \quad f(t)=\frac{1}{2 \pi i} \int_{\mathrm{B}} d s e^{t s} \tilde{f}(s),
$$

the Bromwich path B being a vertical line Res =constant in the half plane of convergence of the Laplace transform. (Very similar equations of motion can be obtained by assuming that the external (laser) field is initially in a number state $N_{0}$, with $N_{0}$ very large [12]. See also the discussion in Section 5.) It is straightforward to obtain

$$
\begin{aligned}
\widetilde{x}(s) & =\frac{1}{s+i \omega_{0}+Q(B, s)} \\
\widetilde{y}_{\boldsymbol{k} \lambda}(s) & =\frac{-i \phi_{\boldsymbol{k} \lambda}\left(s+i \omega_{k}\right)}{\left(s+i \omega_{k}\right)^{2}+B^{2}} \widetilde{x}(s), \\
\widetilde{z}_{\boldsymbol{k} \lambda}(s) & =-\frac{\sqrt{\bar{N}_{0}} \Phi_{\boldsymbol{k}_{0} \lambda_{0}}^{*} \phi_{\boldsymbol{k} \lambda}}{\left(s+i \omega_{k}\right)^{2}+B^{2}} \widetilde{x}(s),
\end{aligned}
$$

with

$$
Q(B, s)=\sum_{\boldsymbol{k}, \lambda}\left|\phi_{\boldsymbol{k} \lambda}\right|^{2} \frac{s+i \omega_{k}}{\left(s+i \omega_{k}\right)^{2}+B^{2}}
$$

and where

$$
B^{2}=\bar{N}_{0}\left|\Phi_{k_{0} \lambda_{0}}\right|^{2}
$$


is proportional to the intensity of the laser field and can be viewed as the "strength" of the observation performed by the laser beam on level \#2 [3]. See the paragraph following Eq. (3.6). Note that the coupling $B$ is related to the Rabi frequency by the simple relation $B=\Omega_{\text {Rabi }} / 2$.

In the continuum limit $(V \rightarrow \infty)$, the matrix elements scale as follows

$$
\lim _{V \rightarrow \infty} \frac{V \omega^{2}}{(2 \pi)^{3}} \sum_{\lambda} \int d \Omega\left|\phi_{\boldsymbol{k} \lambda}\right|^{2} \equiv g^{2} \omega_{0} \chi^{2}(\omega)
$$

where $\Omega$ is the solid angle. The (dimensionless) function $\chi(\omega)$ and coupling constant $g$ have the following general properties, discussed in Appendix A:

$$
\begin{aligned}
\chi^{2}(\omega) & \propto\left\{\begin{array}{ll}
\omega^{2 j \mp 1} & \text { if } \omega \ll \Lambda \\
\omega^{-\beta} & \text { if } \omega \gg \Lambda
\end{array},\right. \\
g^{2} & =\alpha\left(\omega_{0} / \Lambda\right)^{2 j+1 \mp 1},
\end{aligned}
$$

where $j$ is the total angular momentum of the photon emitted in the $2 \rightarrow 1$ transition, $\mp$ represent electric and magnetic transitions, respectively, $\beta(>1)$ is a constant, $\alpha$ the fine structure constant and $\Lambda$ a natural cutoff (of the order of the inverse size of the emitting system, e.g. the Bohr radius for an atom), which determines the range of the atomic or molecular form factor [29].

In order to scale the quantity $B$, we take the limit of very large cavity, by keeping the density of $\Omega_{0}$-photons in the cavity constant:

$$
V \rightarrow \infty, \quad \bar{N}_{0} \rightarrow \infty, \quad \text { with } \quad \frac{\bar{N}_{0}}{V}=n_{0}=\text { const }
$$

and obtain from 3.13

$$
B^{2}=n_{0} V\left|\Phi_{\boldsymbol{k}_{0} \lambda_{0}}\right|^{2}=(2 \pi)^{3} n_{0}\left|\varphi_{\lambda_{0}}\left(\boldsymbol{k}_{0}\right)\right|^{2},
$$

where $\varphi \equiv \Phi V^{1 / 2} /(2 \pi)^{3 / 2}$ is the scaled matrix element of the 1-3 transition. As we shall see, in order to affect significantly the lifetime of level $\# 2$, we shall need a high value of $B$, namely, a laser beam of high intensity. It is therefore interesting to consider a 1-3 transition of the dipole type, in which case the above formula reads

$$
B^{2}=2 \pi \alpha \Omega_{0}\left|\boldsymbol{\epsilon}_{\boldsymbol{k}_{0} \lambda_{0}}^{*} \cdot \boldsymbol{x}_{13}\right|^{2} n_{0},
$$

where $\boldsymbol{x}_{13}$ is the dipole matrix element.

\subsection{Laser off}

Let us first look at the case $B=0$. The laser is off and we expect to recover the well-known physics of the spontaneous emission a two-level system prepared in an excited state and coupled to the vacuum of the radiation field. In this case, $Q(0, s)$ is nothing but the self-energy function

$$
Q(s) \equiv Q(0, s)=\sum_{\boldsymbol{k}, \lambda}\left|\phi_{\boldsymbol{k} \lambda}\right|^{2} \frac{1}{s+i \omega_{k}}
$$




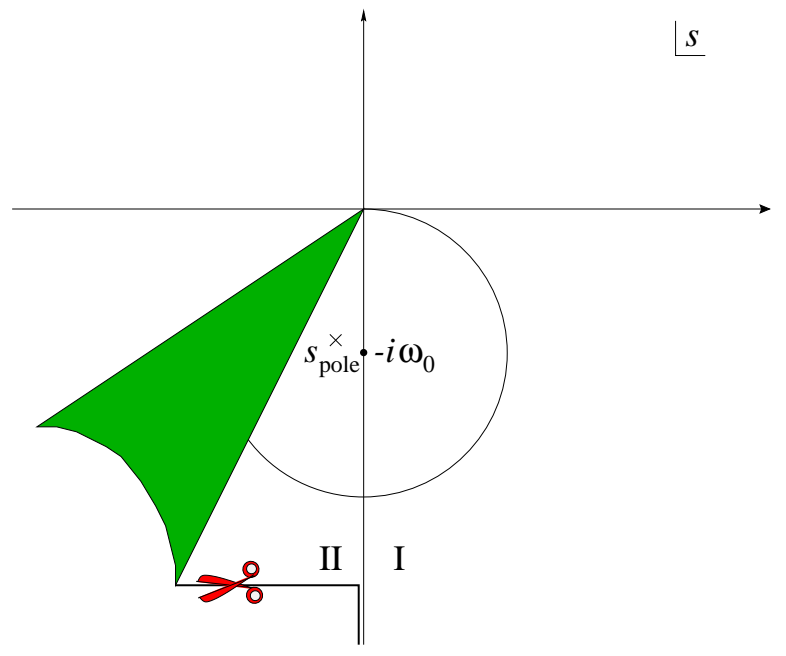

Figure 2: Cut and pole in the $s$-plane $(B=0)$ and convergence circle for the expansion of $Q(s)$ around $s=-i \omega_{0}$. I and II are the first and second Riemann sheets, respectively. The pole is on the second Riemann sheet, at a distance $O\left(g^{2}\right)$ from $-i \omega_{0}$.

which becomes, in the continuum limit,

$$
Q(s) \equiv g^{2} \omega_{0} q(s) \equiv-i g^{2} \omega_{0} \int_{0}^{\infty} d \omega \frac{\chi^{2}(\omega)}{\omega-i s}
$$

where $\chi$ is defined in (3.14). The function $\widetilde{x}(s)$ in Eq. (3.9) (with $B=0$ ) has a logarithmic branch cut, extending from 0 to $-i \infty$, and no singularities on the first Riemann sheet (physical sheet) [22]. On the other hand, it has a simple pole on the second Riemann sheet, that is the solution of the equation

$$
s+i \omega_{0}+g^{2} \omega_{0} q_{\mathrm{II}}(s)=0
$$

where

$$
q_{\mathrm{II}}(s)=q\left(s e^{-2 \pi i}\right)=q(s)+2 \pi \chi^{2}(i s)
$$

is the determination of $q(s)$ on the second Riemann sheet. We note that $g^{2} q(s)$ is $O\left(g^{2}\right)$, so that the pole can be found perturbatively: by expanding $q_{\mathrm{II}}(s)$ around $-i \omega_{0}$ we get a power series, whose radius of convergence is $R_{c}=\omega_{0}$ because of the branch point at the origin. The circle of convergence lies half on the first Riemann sheet and half on the second sheet (Figure 2). The pole is well inside the convergence circle, because $\left|s_{\text {pole }}+i \omega_{0}\right| \sim g^{2} \omega_{0} \ll R_{c}$, and we can write

$$
s_{\text {pole }}=-i \omega_{0}-g^{2} \omega_{0} q_{\mathrm{II}}\left(-i \omega_{0}-0^{+}\right)+O\left(g^{4}\right)=-i \omega_{0}-g^{2} \omega_{0} q\left(-i \omega_{0}+0^{+}\right)+O\left(g^{4}\right),
$$

because $q_{\mathrm{II}}(s)$ is the analytic continuation of $q(s)$ below the branch cut. By using the formula

$$
\lim _{\varepsilon \rightarrow 0^{+}} \frac{1}{x \pm i \varepsilon}=P \frac{1}{x} \mp i \pi \delta(x),
$$


one gets from (3.21)

$$
\begin{aligned}
q\left(-i \eta+0^{+}\right) & =-i \int_{0}^{\infty} d \omega \chi^{2}(\omega) \frac{1}{\omega-\eta-i 0^{+}} \\
& =\pi \chi^{2}(\eta) \theta(\eta)-i P \int_{0}^{\infty} d \omega \chi^{2}(\omega) \frac{1}{\omega-\eta}
\end{aligned}
$$

and by setting

$$
s_{\text {pole }}=-i \omega_{0}+i \Delta E-\frac{\gamma}{2}
$$

one obtains

$$
\gamma=2 \pi g^{2} \omega_{0} \chi^{2}\left(\omega_{0}\right)+O\left(g^{4}\right), \quad \Delta E=g^{2} \omega_{0} P \int_{0}^{\infty} d \omega \frac{\chi^{2}(\omega)}{\omega-\omega_{0}}+O\left(g^{4}\right)
$$

which are the Fermi "golden rule" and the second order correction to the energy of level \#2.

The Weisskopf-Wigner approximation [10] consists in neglecting all branch cut contributions and approximating the self-energy function with a constant (its value in the pole), that is

$$
\widetilde{x}(s)=\frac{1}{s+i \omega_{0}+Q(s)} \simeq \frac{1}{s+i \omega_{0}+Q_{\mathrm{II}}\left(s_{\text {pole }}\right)}=\frac{1}{s-s_{\text {pole }}},
$$

where in the last equality we used the pole equation (3.22). This yields a purely exponential behavior, $x(t)=\exp \left(s_{\text {pole }} t\right)$, without short-time (and long-time) corrections. As is well known, the latter are all contained in the neglected branch cut contribution.

\subsection{Laser on}

We turn now our attention to the situation with the laser switched on $(B \neq 0)$ and tuned at the 1-3 transition frequency $\Omega_{0}$. The self energy function $Q(B, s)$ in (3.12) depends on $B$ and can be written in terms of the self energy function $Q(s)$ in absence of laser field [Eq. (3.20)], by making use of the following remarkable property:

$Q(B, s)=\frac{1}{2} \sum_{\boldsymbol{k}, \lambda}\left|\phi_{\boldsymbol{k} \lambda}\right|^{2}\left(\frac{1}{s+i \omega_{k}+i B}+\frac{1}{s+i \omega_{k}-i B}\right)=\frac{1}{2}[Q(s+i B)+Q(s-i B)]$.

Notice, incidentally, that in the continuum limit $(V \rightarrow \infty)$, due to the above formula, $Q(B, s)$ scales just like $Q(s)$. The position of the pole $s_{\text {pole }}$ (and as a consequence the lifetime $\left.\tau_{\mathrm{E}} \equiv \gamma^{-1}=-1 / 2 \operatorname{Re} s_{\text {pole }}\right)$ depends on the value of $B$. There are now two branch cuts in the complex $s$ plane, due to the two terms in $(3.30)$. They lie over the imaginary axis, along $(-i \infty,-i B]$ and $(-i \infty,+i B]$.

The pole satisfies the equation

$$
s+i \omega_{0}+Q(B, s)=0
$$



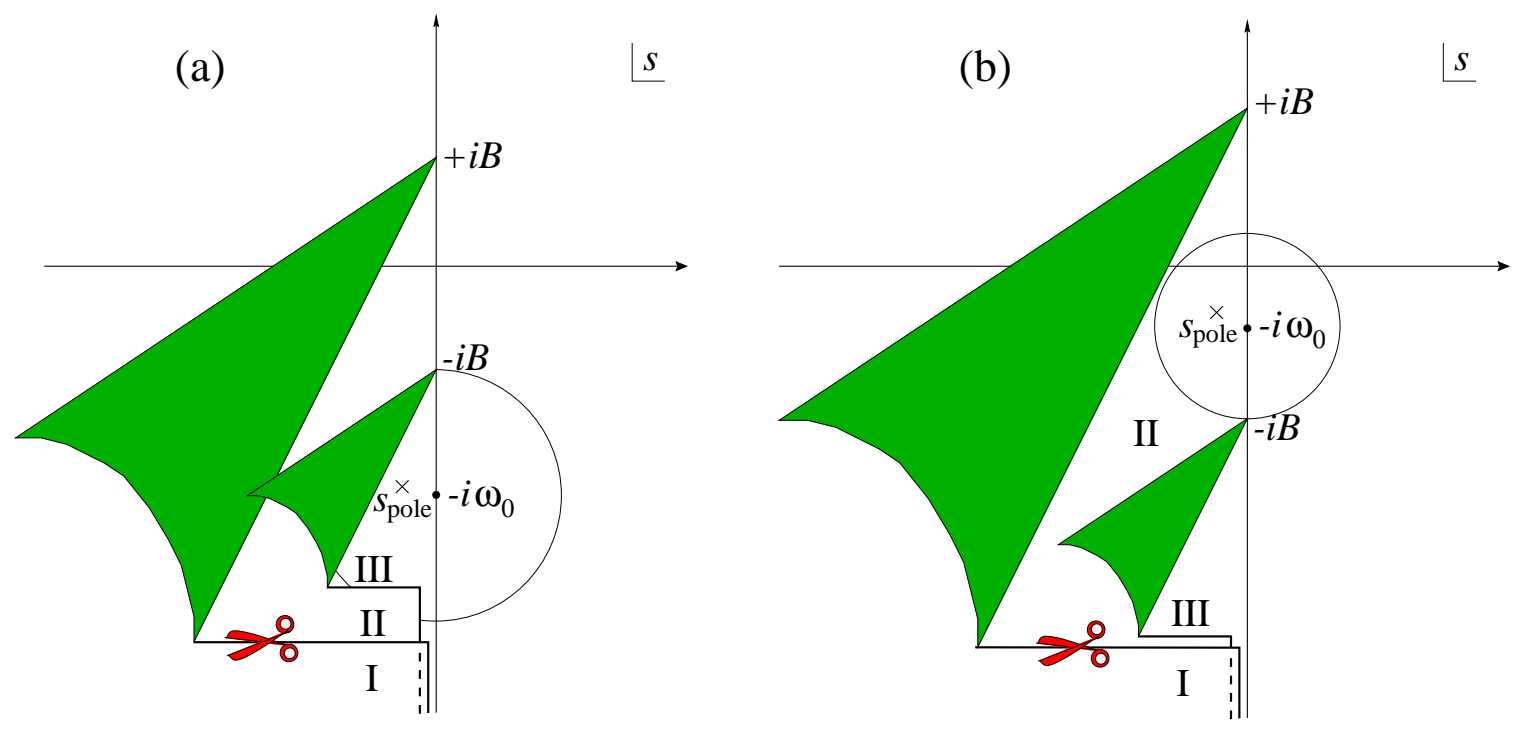

Figure 3: Cuts and pole in the $s$-plane $(B \neq 0)$ and convergence circle for the expansion of $Q(B, s)$ around $s=-i \omega_{0}$. I , II and III are the first, second and third Riemann sheets, respectively. (a) $B<\omega_{0}$. (b) $B>\omega_{0}$. In both cases, the pole is at a distance $O\left(g^{2}\right)$ from $-i \omega_{0}$.

where $Q(B, s)$ is of order $g^{2}$, as before, and can again be expanded in power series around $s=-i \omega_{0}$, in order to find the pole perturbatively. However, this time one has to choose the right determination of the function $Q(B, s)$. Two cases are mathematically possible: a) The branch point $-i B$ is situated above $-i \omega_{0}$, so that $-i \omega_{0}$ lies on both cuts. See Figure $3(\mathrm{a})$; b) The branch point $-i B$ is situated below $-i \omega_{0}$, so that $-i \omega_{0}$ lies only on the upper branch cut. See Figure $3(\mathrm{~b})$. We notice that, although mathematically conceivable, the latter case $\left(B>\omega_{0}\right)$ cannot be tackled within our approximations, for a number of additional effects would then have to be considered: multi-photon processes would take place, the other atomic levels would start to play an important role and our approach (3-level atom in the rotating wave approximation) would no longer be valid. We therefore restrict our attention to values of $B$ that are high (of the same order of magnitude as those utilized in electromagnetic induced transparency), but not extremely high, so that our starting approximations still apply.

In case a), i.e. for $B<\omega_{0}$, the pole is on the third Riemann sheet (under both cuts) and the power series converges in a circle lying half on the first and half on the third Riemann sheet, within a convergence radius $R_{c}=\omega_{0}-B$, which decreases as $B$ increases [Figure [3(a)]. For the sake of completeness we also notice that in case b), i.e. for $B>\omega_{0}$, the pole would be on the second Riemann sheet (under the upper cut only) and the power series would converge in a circle lying half on the first and half on the second Riemann sheet, within a convergence radius $R_{c}=B-\omega_{0}$, which increases with $B$ [Figure $3(\mathrm{~b})]$. 
In either cases we can write, for $\left|s_{\text {pole }}+i \omega_{0}\right|<R_{c}=\left|B-\omega_{0}\right|$,

$$
\begin{aligned}
s_{\text {pole }} & =-i \omega_{0}-\frac{1}{2}\left\{Q\left[-i\left(\omega_{0}+B\right)+0^{+}\right]+Q\left[-i\left(\omega_{0}-B\right)+0^{+}\right]\right\}+O\left(g^{4}\right) \\
& =-i \omega_{0}-\frac{1}{2} g^{2} \omega_{0}\left\{q\left[-i\left(\omega_{0}+B\right)+0^{+}\right]+q\left[-i\left(\omega_{0}-B\right)+0^{+}\right]\right\}+O\left(g^{4}\right) .
\end{aligned}
$$

Equation (3.32) enables us to analyze the temporal behavior of state \#2.

\subsection{Decay rate vs $B$}

We write, as in (3.27),

$$
s_{\text {pole }}=-i \omega_{0}+i \Delta E(B)-\frac{\gamma(B)}{2}
$$

Substituting (3.26) into (3.32) and taking the real part, one obtains the following expression for the decay rate

$$
\gamma(B)=\pi g^{2} \omega_{0}\left[\chi^{2}\left(\omega_{0}+B\right)+\chi^{2}\left(\omega_{0}-B\right) \theta\left(\omega_{0}-B\right)\right]+O\left(g^{4}\right) .
$$

On the other hand, by (3.28), one can write

$$
\gamma(B)=\gamma \frac{\chi^{2}\left(\omega_{0}+B\right)+\chi^{2}\left(\omega_{0}-B\right) \theta\left(\omega_{0}-B\right)}{2 \chi^{2}\left(\omega_{0}\right)}+O\left(g^{4}\right) .
$$

This is the central result of this paper and involves no approximations: Equation (3.35) expresses the "new" lifetime $\gamma(B)^{-1}$, when the system is bathed in an intense laser field $B$, in terms of the "ordinary" lifetime $\gamma^{-1}$, when there is no laser field. By taking into account the general behavior (3.15) of the matrix elements $\chi^{2}(\omega)$ and substituting into (3.35), one gets to $O\left(g^{4}\right)$

$$
\gamma(B) \simeq \frac{\gamma}{2}\left[\left(1+\frac{B}{\omega_{0}}\right)^{2 j \mp 1}+\left(1-\frac{B}{\omega_{0}}\right)^{2 j \mp 1} \theta\left(\omega_{0}-B\right)\right], \quad(B \ll \Lambda)
$$

where $\mp$ refers to 1-2 transitions of electric and magnetic type, respectively. Observe that, since $\Lambda \sim$ inverse Bohr radius, only the case $B<\omega_{0} \ll \Lambda$ is the physically relevant one [12]. The decay rate is profoundly modified by the presence of the laser

field. Its behavior is shown in Figure 4 for a few values of $j$. In general, for $j>1$ (1-2 transitions of electric quadrupole, magnetic dipole or higher), the decay rate $\gamma(B)$ increases with $B$, so that the lifetime $\gamma(B)^{-1}$ decreases as $B$ is increased. If one looks at $B$ as the strength of the "observation" performed by the laser beam on level \#2 [3], one can view this phenomenon as an "inverse" quantum Zeno effect, for decay is enhanced (rather than suppressed) by observation.

As we shall see in Sections 1 and 5, the emitted photons have different frequencies [for they correspond to decay onto different dressed (Fano) states]. By selecting the 

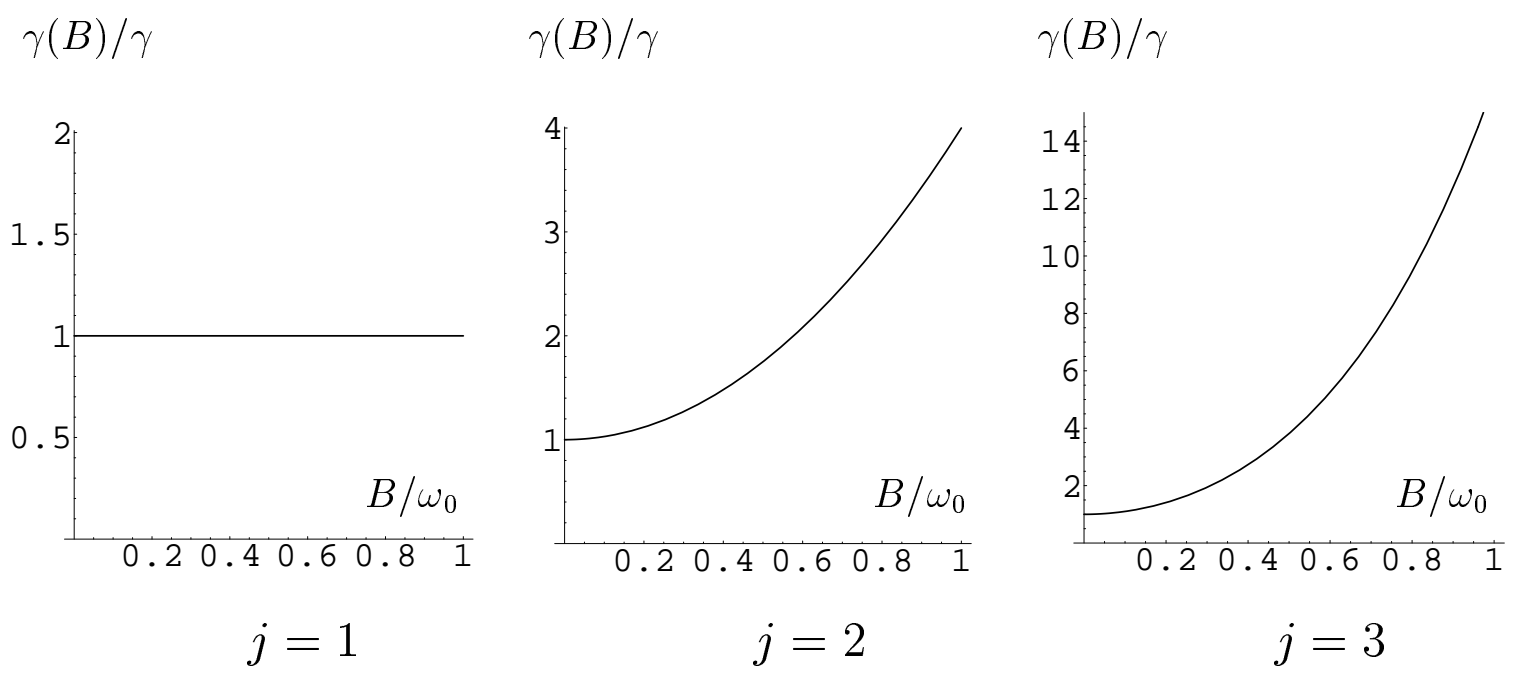

Figure 4: The decay rate $\gamma(B)$ vs $B$, for electric transitions with $j=1,2,3 ; \gamma(B)$ is in units $\gamma$ and $B$ in units $\omega_{0}$. Notice the different scales on the vertical axis.

photon wavelength (i.e., by means of filters), one could therefore also measure the different contributions to the inverse lifetime in (3.35)-(3.36). We shall come back to this point later.

As already emphasized, Eq. (3.36) is valid for $B \ll \Lambda$. In the opposite (unphysical) case $B \gg \Lambda$, by (3.15) and (3.35), one gets to $\mathrm{O}\left(g^{4}\right)$

$$
\gamma(B) \simeq \frac{\gamma}{2} \frac{\chi^{2}(B)}{\chi^{2}\left(\omega_{0}\right)} \propto(B / \Lambda)^{-\beta} . \quad(B \gg \Lambda)
$$

This result is similar to that obtained in Ref. [3]. If such high values of $B$ were experimentally obtainable, the decay would be considerably hindered and $B$ could be properly viewed as the "strength" of the observation performed by the laser field on level \#2 (quantum Zeno effect). However, in such a case, many additional effects would have to be considered and our analysis should be modified in order to take them into account. A similar remark was made by Kofman and Kurizki in a different context 13 .

A final remark is now in order. If one would use the approximation (3.29) in Eq. (3.30), in order to evaluate the new lifetime, i.e. if one sets $Q(s)=Q\left(s_{\text {pole }}\right)=$ const, one would obtain $Q(B, s)=Q(s)=Q\left(s_{\text {pole }}\right)$, i.e. no $B$-dependence. Therefore, the effect we are discussing is ultimately due to the nonexponential contributions arising from the cut. In particular, viewed from the perspective of the time domain, this effect is ascribable to the quadratic short-time behavior of the $2 \rightarrow 1$ decay.

\subsection{Estimates}

We saw in the previous subsection that the ratio $B / \omega_{0}$ is the relevant quantity in the evaluation of the modified lifetime. Let us therefore try to get a rough feeling for the magnitude of the relevant physical parameters. In order to affect significantly the 
lifetime of level $\# 2$, we have to look at rather large values of $B$ : for instance at 1-3 transition of the electric dipole type. In such a case, Eq. (3.19) applies:

$$
B^{2}=2 \pi \alpha \Omega_{0}\left|\boldsymbol{\epsilon}_{\boldsymbol{k}_{0} \lambda_{0}}^{*} \cdot \boldsymbol{x}_{13}\right|^{2} n_{0} .
$$

Considering the angle average

$$
\left\langle\left|\epsilon_{\boldsymbol{k}_{0} \lambda_{0}}^{*} \cdot \boldsymbol{x}_{13}\right|^{2}\right\rangle=\frac{1}{3}\left|\boldsymbol{x}_{13}\right|^{2}
$$

and remembering that the decay rate is

$$
\Gamma_{13}=\frac{4}{3} \alpha\left|\boldsymbol{x}_{13}\right|^{2} \Omega_{0}^{3},
$$

we obtain

$$
B^{2}=\frac{\pi}{2} n_{0} \frac{\Gamma_{13}}{\Omega_{0}^{2}}
$$

which, reinserting c's and $\hbar$ 's, reads

$$
B^{2}=\frac{\pi}{2} n_{0} \hbar \Omega_{0} \frac{c^{3}}{\Omega_{0}^{3}} \hbar \Gamma_{13}=\left(n_{0} \hbar \Omega_{0}\right) \frac{\lambda_{L}^{3}}{16 \pi^{2}}\left(\hbar \Gamma_{13}\right),
$$

where $\lambda_{L}=2 \pi c / \Omega_{0}$. The quantity $B^{2}$ has dimensions of squared energy and is given by the product of the energy of the laser field contained in the volume $\lambda_{L}^{3} / 16 \pi^{2}$ times the energy spread of the $1-3$ transition $\Omega_{0}$. Therefore $B$ depends on both laser and atomic system. Observe that $n_{0} \lambda_{L}^{3}$ is the number of laser photons contained in the volume $\lambda_{L}^{3}$.

In terms of laser power $P$ and laser spot area $A$, Eq. (3.42) reads

$$
B^{2}=\frac{P}{c A} \frac{\lambda_{L}^{3}}{16 \pi^{2}}\left(\hbar \Gamma_{13}\right)=132 \frac{P \lambda_{L}^{3}}{A}\left(\hbar \Gamma_{13}\right) \mathrm{eV}^{2},
$$

where $P$ is expressed in Watt, $\lambda_{L}$ in $\mu \mathrm{m}, A$ in $\mu \mathrm{m}^{2}$ and $\hbar \Gamma$ in $\mathrm{eV}$. In Eq. (3.43) the quantity $B$ is expressed in suitable units and can be easily compared to $\omega_{0}$ [the ratio $B / \omega_{0}$ being the relevant quantity in Eq. (3.36)]. For laser intensities that are routinely used in the study of electromagnetic induced transparency, the effect should be experimentally observable. For a quick comparison remember that $B$ is just half the Rabi frequency of the resonant transition $1-3$ [see paragraph following Eq. (3.13)].

\section{Photon spectrum}

It is interesting to look at the spectrum of the emitted photons. It is easy to check that, in the Weisskopf-Wigner approximation, the survival probability $|\langle\psi(0) \mid \psi(t)\rangle|^{2}=|x(t)|^{2}$ decreases exponentially with time. The standard way to 
$d P / d \omega$

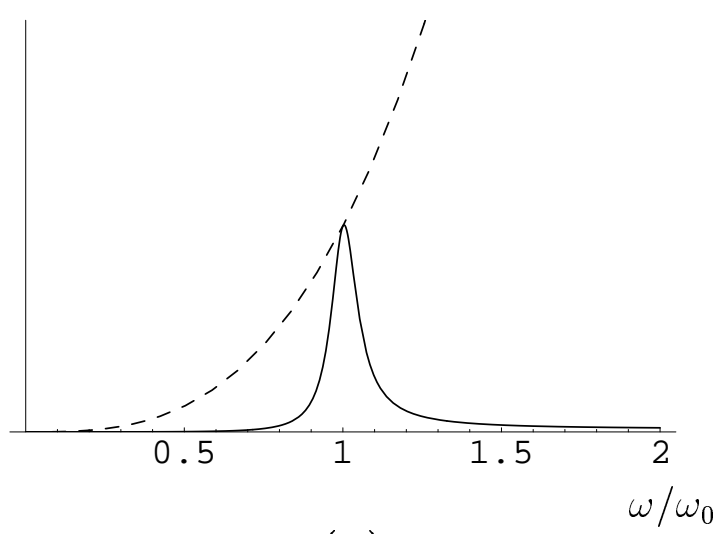

(a) $d P_{B} / d \omega$

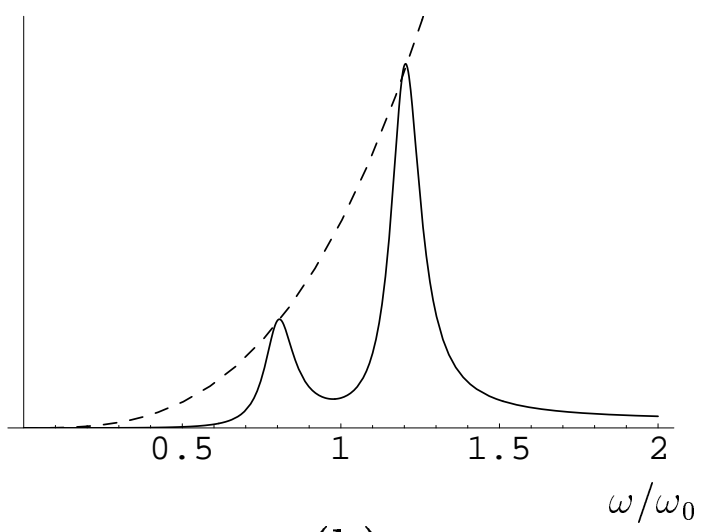

(b)

Figure 5: The spectrum (4.5) of the emitted photons. The height of the Lorentzians is proportional to the matrix element $\chi^{2}(\omega)$ (dashed line). We chose an electric quadrupole transition, with $j=2$ and $\gamma=10^{-1} \bar{\omega}_{0}$, and used arbitrary units on the vertical axis. a) $\mathrm{B}=0$; b) $B=\bar{\omega}_{0} / 5$; note that from $(3.36) \gamma(B)=(28 / 25) \gamma$.

obtain this result is to neglect the cut contribution in the complex $s$ plane, or equivalently, to substitute in (3.9) the pole determination of the self-energy function:

$$
\widetilde{x}(s)=\frac{1}{s+i \omega_{0}+Q(B, s)} \approx \frac{1}{s+i \omega_{0}+Q\left(B, s_{\text {pole }}\right)}=\frac{1}{s-s_{\text {pole }}},
$$

from which one gets

$$
x(t)=\exp \left(s_{\text {pole }} t\right)=\exp \left(-i \bar{\omega}_{0} t-\frac{\gamma(B)}{2} t\right),
$$

where $\bar{\omega}_{0}=\omega_{0}-\Delta E(B)$. In this approximation, for any value of $B$, the spectrum of the emitted photons is Lorentzian. The proof is straightforward and is given in Appendix B. One finds that, for $B=0$, the probability to emit a photon in the range $(\omega, \omega+d \omega)$ reads

$$
d P_{B=0}=g^{2} \omega_{0} \chi^{2}(\omega) f_{L}\left(\omega-\bar{\omega}_{0} ; \gamma\right) d \omega
$$

where

$$
f_{L}(\omega ; \gamma)=\frac{1}{\omega^{2}+\gamma^{2} / 4}
$$

On the other hand, when $B \neq 0$ one gets:

$$
d P_{B}=g^{2} \omega_{0} \chi^{2}(\omega) \frac{1}{2}\left[f_{L}\left(\omega-\bar{\omega}_{0}-B ; \gamma(B)\right)+f_{L}\left(\omega-\bar{\omega}_{0}+B ; \gamma(B)\right)\right] d \omega .
$$

The emission probability is given by the sum of two Lorentzians, centered in $\bar{\omega}_{0} \pm B$. We see that the emission probability of a photon of frequency $\bar{\omega}_{0}+B\left(\bar{\omega}_{0}-B\right)$ 
increases (decreases) with $B$ (Figure 5). The linewidths are modified according to Eq. (3.36). When $B$ reaches the "threshold" value $\bar{\omega}_{0}$, only the photon of higher frequency $\left(\bar{\omega}_{0}+B\right)$ is emitted (with increasing probability vs $B$ ).

Photons of different frequencies are therefore emitted with different rates. We shall understand better the features of the emission in the next section, by looking at the dressed states of the system.

\section{Dressed states and links with induced trans- parency}

It is useful and interesting to look at our results from a different perspective, by analyzing the modifications of the energy levels provoked by the laser field. For simplicity, since the average number $\bar{N}_{0}$ of $\boldsymbol{k}_{0}$-photons in the total volume $V$ can be considered very large, we shall perform our analysis in terms of number (rather than coherent) states of the electromagnetic field. In this limit,

$$
\left\langle 1 ; 0_{\boldsymbol{k} \lambda}, N_{0}\left|H_{\mathrm{int}}\right| 3 ; 0_{\boldsymbol{k} \lambda}, N_{0}-1\right\rangle=\sqrt{N_{0}} \Phi_{\boldsymbol{k}_{0} \lambda_{0}} \gg\left\langle 1 ; 1_{\boldsymbol{k} \lambda}, N_{0}-1\left|H_{\mathrm{int}}\right| 3 ; 0_{\boldsymbol{k} \lambda}, N_{0}-1\right\rangle=\Phi_{\boldsymbol{k} \lambda},
$$

$\forall(\boldsymbol{k}, \lambda) \neq\left(\boldsymbol{k}_{0}, \lambda_{0}\right)$. [This is equivalent to (2.6).] In the above equation and henceforth, the vector $\left|i ; n_{\boldsymbol{k} \lambda}, M_{0}\right\rangle$ represents an atom in state $|i\rangle$, with $n_{\boldsymbol{k} \lambda}(\boldsymbol{k}, \lambda)$-photons and $M_{0}$ laser photons.

In the above approximation, the Hamiltonian (2.1) becomes

$$
\begin{aligned}
H \simeq & \omega_{0}|2\rangle\left\langle 2\left|+\Omega_{0}\right| 3\right\rangle\langle 3|+\sum_{\boldsymbol{k}, \lambda} \omega_{k} a_{\boldsymbol{k} \lambda}^{\dagger} a_{\boldsymbol{k} \lambda}+\sum_{\boldsymbol{k}, \lambda}^{\prime}\left(\phi_{\boldsymbol{k} \lambda} a_{\boldsymbol{k} \lambda}^{\dagger}|1\rangle\left\langle 2\left|+\phi_{\boldsymbol{k} \lambda}^{*} a_{\boldsymbol{k} \lambda}\right| 2\right\rangle\langle 1|\right) \\
& +\left(\Phi_{\boldsymbol{k}_{0} \lambda_{0}} a_{\boldsymbol{k}_{0} \lambda_{0}}^{\dagger}|1\rangle\left\langle 3\left|+\Phi_{\boldsymbol{k}_{0} \lambda_{0}}^{*} a_{\boldsymbol{k}_{0} \lambda_{0}}\right| 3\right\rangle\langle 1|\right),
\end{aligned}
$$

where a prime means that the summation does not include $\left(\boldsymbol{k}_{0}, \lambda_{0}\right)$ [due to hypothesis (2.3)]. Besides (2.8), there is now another conserved quantity: indeed the operator

$$
\mathcal{N}_{0}=|3\rangle\langle 3|+a_{\boldsymbol{k}_{0} \lambda_{0}}^{\dagger} a_{\boldsymbol{k}_{0} \lambda_{0}}
$$

satisfies

$$
\left[H, \mathcal{N}_{0}\right]=\left[\mathcal{N}_{0}, \mathcal{N}\right]=0 .
$$

In this case, the system evolves in the subspace labelled by the two eigenvalues $\mathcal{N}=1$ and $\mathcal{N}_{0}=N_{0}$, whose states read

$$
|\psi(t)\rangle=x(t)\left|2 ; 0, N_{0}\right\rangle+\sum_{\boldsymbol{k}, \lambda}^{\prime} y_{\boldsymbol{k} \lambda}(t)\left|1 ; 1_{\boldsymbol{k} \lambda}, N_{0}\right\rangle+\sum_{\boldsymbol{k}, \lambda}^{\prime} z_{\boldsymbol{k} \lambda}(t)\left|3 ; 1_{\boldsymbol{k} \lambda}, N_{0}-1\right\rangle .
$$

By using the Hamiltonian (5.2) and the states (5.5) and identifying $N_{0}$ with $\bar{N}_{0}=$ $\left|\alpha_{0}\right|^{2}$, the Schrödinger equation yields again the equations of motion (3.4), obtained by assuming a coherent state for the laser mode. Our analysis is therefore independent of the statistics of the driving field, provided it is sufficiently intense, and the (convenient) use of number states is completely justified. 
Energy conservation implies that if there are two emitted photons with different energies (as we saw in the previous section), there are two levels of different energies to which the atom can decay. This can be seen by considering the laser-dressed (Fano) atomic states [30]. The shift of the dressed states can be obtained directly from the structure of the Hamiltonian (5.2). In the sector $\mathcal{N}_{0}=N_{0}$, the operator $\mathcal{N}_{0}$ is proportional to the unit operator, the constant of proportionality being its eigenvalue. Hence one can write the Hamiltonian in the following form

$$
H=H-\Omega_{0} \mathcal{N}_{0}+\Omega_{0} N_{0},
$$

which, by the setting $E_{1}+N_{0} \Omega_{0}=0$, reads

$$
\begin{aligned}
H= & H_{0}+H_{\mathrm{int}} \\
= & \omega_{0}|2\rangle\langle 2|+\sum_{\boldsymbol{k}, \lambda}^{\prime} \omega_{k} a_{\boldsymbol{k} \lambda}^{\dagger} a_{\boldsymbol{k} \lambda}+\sum_{\boldsymbol{k}, \lambda}^{\prime}\left(\phi_{\boldsymbol{k} \lambda} a_{\boldsymbol{k}_{\lambda}}^{\dagger}|1\rangle\left\langle 2\left|+\phi_{\boldsymbol{k} \lambda}^{*} a_{\boldsymbol{k} \lambda}\right| 2\right\rangle\langle 1|\right) \\
& +\left(\Phi_{\boldsymbol{k}_{0} \lambda_{0}} a_{\boldsymbol{k}_{0} \lambda_{0}}^{\dagger}|1\rangle\left\langle 3\left|+\Phi_{\boldsymbol{k}_{0} \lambda_{0}}^{*} a_{\boldsymbol{k}_{0} \lambda_{0}}\right| 3\right\rangle\langle 1|\right) .
\end{aligned}
$$

On the other hand, in the sector $\mathcal{H}_{\mathcal{N} \mathcal{N}_{0}}$ with $\mathcal{N}=1$ and $\mathcal{N}_{0}=N_{0}$, the last term becomes

$$
\left(\Phi_{\boldsymbol{k}_{0} \lambda_{0}} a_{\boldsymbol{k}_{0} \lambda_{0}}^{\dagger}|1\rangle\left\langle 3\left|+\Phi_{\boldsymbol{k}_{0} \lambda_{0}}^{*} a_{\boldsymbol{k}_{0} \lambda_{0}}\right| 3\right\rangle\langle 1|\right)=\left(\Phi_{\boldsymbol{k}_{0} \lambda_{0}} \sqrt{N_{0}}|1\rangle\left\langle 3\left|+\Phi_{\boldsymbol{k}_{0} \lambda_{0}}^{*} \sqrt{N_{0}}\right| 3\right\rangle\langle 1|\right) .
$$

Let us diagonalize this operator, i.e. let us look for two non-interacting states $|+\rangle$ and $|-\rangle$ which are linear combinations of the old states $|1\rangle$ and $|3\rangle$. To this end we write

$$
\begin{aligned}
& |1\rangle=\frac{1}{\sqrt{2}}\left(|+\rangle+e^{i \delta}|-\rangle\right), \\
& |3\rangle=\frac{e^{i \alpha}}{\sqrt{2}}\left(|+\rangle-e^{i \delta}|-\rangle\right),
\end{aligned}
$$

with $|+\rangle$ e $|-\rangle$ orthonormal:

$$
\langle+\mid+\rangle=\langle-\mid-\rangle=1, \quad\langle+\mid-\rangle=0 .
$$

Plugging (5.9) into (5.7), the interaction term becomes

$$
\begin{aligned}
H_{\mathrm{int}}= & \sum_{\boldsymbol{k}, \lambda}^{\prime}\left[\left(\frac{\phi_{\boldsymbol{k} \lambda}}{\sqrt{2}} a_{\boldsymbol{k} \lambda}^{\dagger}|+\rangle\left\langle 2\left|+\frac{\phi_{\boldsymbol{k} \lambda}^{*}}{\sqrt{2}} a_{\boldsymbol{k} \lambda}\right| 2\right\rangle\langle+|\right)\right. \\
& \left.+\left(\frac{\phi_{\boldsymbol{k} \lambda}}{\sqrt{2}} e^{i \delta} a_{\boldsymbol{k} \lambda}^{\dagger}|-\rangle\left\langle 2\left|+\frac{\phi_{\boldsymbol{k} \lambda}^{*}}{\sqrt{2}} e^{-i \delta} a_{\boldsymbol{k} \lambda}\right| 2\right\rangle\langle-|\right)\right] \\
& +B\left[\frac{e^{i \beta}}{2} e^{-i \alpha}\left(|+\rangle+e^{i \delta}|-\rangle\right)\left(\langle+|-e^{-i \delta}\langle-|\right)+\text { h.c. }\right],
\end{aligned}
$$

where we have set $\Phi_{\boldsymbol{k}_{0} \lambda_{0}} \sqrt{N_{0}}=B e^{i \beta}$. Rearranging the last term

$$
B\left[\frac{e^{i(\beta-\alpha)}}{2}\left(|+\rangle\langle+|-|-\rangle\left\langle-\left|+e^{i \delta}\right|-\right\rangle\left\langle+\left|-e^{-i \delta}\right|+\right\rangle\langle-|\right)\right.
$$




$$
\begin{gathered}
\left.+\frac{e^{-i(\beta-\alpha)}}{2}\left(|+\rangle\langle+|-|-\rangle\left\langle-\left|+e^{-i \delta}\right|+\right\rangle\left\langle-\left|-e^{i \delta}\right|-\right\rangle\langle+|\right)\right] \\
=B \cos (\beta-\alpha)(|+\rangle\langle+|-|-\rangle\langle-|)+i B \sin (\beta-\alpha)\left(e^{i \delta}|-\rangle\left\langle+\left|-e^{-i \delta}\right|+\right\rangle\langle-|\right)
\end{gathered}
$$

and setting $\alpha=\beta$ the two states $|+\rangle$ and $|-\rangle$ decouple and one gets

$$
\begin{aligned}
H_{\mathrm{int}}= & \sum_{\boldsymbol{k}, \lambda}^{\prime}\left[\left(\frac{\phi_{\boldsymbol{k} \lambda}}{\sqrt{2}} a_{\boldsymbol{k} \lambda}^{\dagger}|+\rangle\left\langle 2\left|+\frac{\phi_{\boldsymbol{k} \lambda}^{*}}{\sqrt{2}} a_{\boldsymbol{k} \lambda}\right| 2\right\rangle\langle+|\right)\right. \\
& \left.+\left(\frac{\phi_{\boldsymbol{k} \lambda}}{\sqrt{2}} e^{i \delta} a_{\boldsymbol{k} \lambda}^{\dagger}|-\rangle\left\langle 2\left|+\frac{\phi_{\boldsymbol{k} \lambda}^{*}}{\sqrt{2}} e^{-i \delta} a_{\boldsymbol{k} \lambda}\right| 2\right\rangle\langle-|\right)\right] \\
& +B|+\rangle\langle+|-B|-\rangle\langle-| .
\end{aligned}
$$

Therefore we can write

$$
H_{0}+H_{\mathrm{int}}=H_{0}^{\prime}+H_{\mathrm{int}}^{\prime},
$$

where the primed free and interaction Hamiltonians read respectively

$$
\begin{aligned}
H_{0}^{\prime} & =\omega_{0}|2\rangle\langle 2|+B|+\rangle\langle+|-B|-\rangle\langle-|+\sum_{\boldsymbol{k}, \lambda}^{\prime} \omega_{k} a_{\boldsymbol{k} \lambda}^{\dagger} a_{\boldsymbol{k} \lambda}, \\
H_{\mathrm{int}}^{\prime} & =\sum_{\boldsymbol{k}, \lambda}^{\prime}\left[\left(\frac{\phi_{\boldsymbol{k} \lambda}}{\sqrt{2}} a_{\boldsymbol{k} \lambda}^{\dagger}|+\rangle\left\langle 2\left|+\frac{\phi_{\boldsymbol{k} \lambda}^{*}}{\sqrt{2}} a_{\boldsymbol{k} \lambda}\right| 2\right\rangle\langle+|\right)+\left(\frac{\phi_{\boldsymbol{k} \lambda}}{\sqrt{2}} a_{\boldsymbol{k} \lambda}^{\dagger}|-\rangle\left\langle 2\left|+\frac{\phi_{\boldsymbol{k} \lambda}^{*}}{\sqrt{2}} a_{\boldsymbol{k} \lambda}\right| 2\right\rangle\langle-|\right)\right]
\end{aligned}
$$

and we set $\delta=0$. We see that the laser dresses the states $|1\rangle$ and $|3\rangle$, which (if one includes the $\Omega_{0}$ photon) are degenerate [with energy $E=0$, due to the choice of the zero of energy: see line after (5.6)]. The dressed states $|+\rangle$ and $|-\rangle$ have energies $+B$ and $-B$ and interact with state $|2\rangle$ with a coupling $\phi_{\boldsymbol{k} \lambda} / \sqrt{2}$. Since $2 B=\Omega_{\text {Rabi }}$ these are the well-known Autler-Townes doublet [31].

Therefore, by applying the Fermi golden rule, the decay rates into the dressed states read

$$
\gamma_{+}=2 \pi g^{2} \omega_{0} \frac{\chi^{2}\left(\omega_{0}-B\right)}{2} \quad \gamma_{-}=2 \pi g^{2} \omega_{0} \frac{\chi^{2}\left(\omega_{0}+B\right)}{2}
$$

and the total decay rate of state $|2\rangle$ is given by their sum

$$
\gamma=\gamma_{+}+\gamma_{-},
$$

which yields (3.34). One sees why there is a threshold at $B=\omega_{0}$ : For $B<\omega_{0}$, the energies of both dressed states $| \pm\rangle$ is lower than that of the initial state $|2\rangle$ [Figure 6(a)]. The decay rate $\gamma_{-}$increases with $B$, whereas $\gamma_{+}$decreases with $B$; their sum $\gamma$ increases with $B$. These two decays (and their lifetimes) could be easily distinguished by selecting the frequencies of the emitted photons, e.g. by means of filters.

We also notice, for completeness, that when $B>\omega_{0}$, the energy of the dressed state $|+\rangle$ is larger than that of state $|2\rangle$ and this decay channel disappears [Figure 6(b)]. 


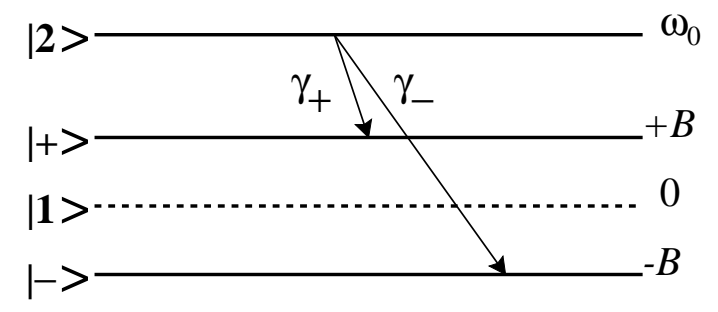

(a)

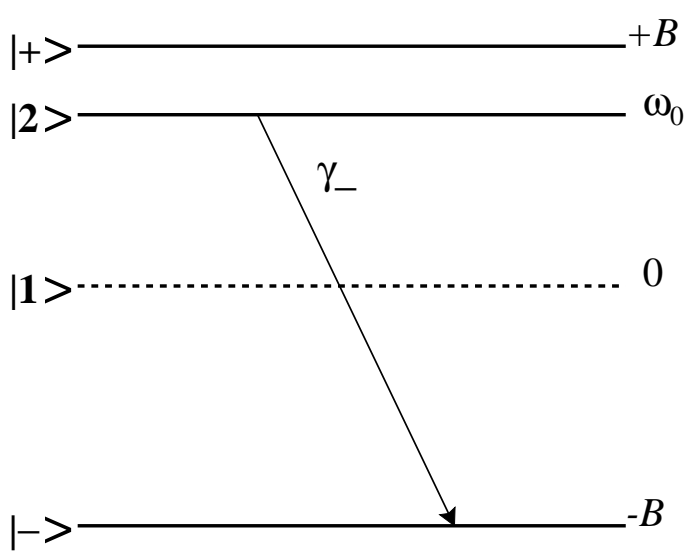

(b)

Figure 6: Shift of the dressed states $|+\rangle$ and $|-\rangle$ vs $B$. (a) For $B<\omega_{0}$ there are two decay channels, with $\gamma_{-}>\gamma_{+}$. (b) For $B>\omega_{0}$ level $|+\rangle$ is above level $|2\rangle$ and only the $\gamma_{-}$decay channel remains.

As repeatedly emphasized, this situation is unphysical and would require a different analysis, for additional effects would play an important role.

Finally, let us emphasize that if state $|2\rangle$ were below state $|1\rangle$, our system would become a three-level system in a ladder configuration, and the shift of the dressed states would give rise to electromagnetically induced transparency [26]. The situation we consider and the laser power required to bring these effects to light are therefore similar to those used in induced transparency.

\section{Influence of other levels}

Let us now see how our results are modified by the presence of off-resonant levels. To this end we generalize the three-level Hamiltonian (2.7) by including other offresonant levels $|j\rangle(j=4, \cdots, N)$ in our analysis:

$$
\begin{aligned}
H= & \omega_{0}|2\rangle\left\langle 2\left|+\sum_{j=3}^{N} \Omega_{j}\right| j\right\rangle\langle j|+\sum_{\boldsymbol{k}, \lambda} \omega_{k} a_{\boldsymbol{k} \lambda}^{\dagger} a_{\boldsymbol{k} \lambda}+\sum_{\boldsymbol{k}, \lambda}^{\prime}\left(\phi_{\boldsymbol{k} \lambda} a_{\boldsymbol{k} \lambda}^{\dagger}|1\rangle\left\langle 2\left|+\phi_{\boldsymbol{k} \lambda}^{*} a_{\boldsymbol{k} \lambda}\right| 2\right\rangle\langle 1|\right) \\
& +\sum_{j=3}^{N}\left(\Phi_{j} \alpha_{0}^{*} e^{i \Omega_{3} t}|1\rangle\left\langle j\left|+\Phi_{j}^{*} \alpha_{0} e^{-i \Omega_{3} t}\right| j\right\rangle\langle 1|\right)
\end{aligned}
$$

where $\Phi_{j}=\Phi_{j, \boldsymbol{k}_{0} \lambda_{0}}$ are the matrix elements of the $1 \leftrightarrow j$ transitions and $\Omega_{j}=E_{j}-E_{1}$ the energy of level $|j\rangle$ [in particular, $\Phi_{3, \boldsymbol{k}_{0} \lambda_{0}}=\Phi_{\boldsymbol{k}_{0} \lambda_{0}}$ and $\Omega_{3}=\Omega_{0}$ in Eq. (2.7)].

By a calculation similar to that used in Section 3 one gets again the expression (3.9) for the Laplace trasform of the survival amplitude, with the new self-energy 
function modified by the presence of other levels

$$
Q(B, s)=\sum_{\boldsymbol{k}, \lambda} \frac{\left|\phi_{\boldsymbol{k} \lambda}\right|^{2}}{s+i \omega_{k}+B^{2} \sum_{j=3}^{N} \frac{f_{j}}{s+i \delta_{j}+i \omega_{k}}},
$$

where $f_{j}=\left|\Phi_{j}\right|^{2} /\left|\Phi_{3}\right|^{2}$ and $\delta_{j}=\Omega_{j}-\Omega_{3}$.

The denominator of $Q(B, s)$ is now a polynomial of order $N-1$ (when $N=3$ one reobtains Eq. (3.12) with a quadratic polynomial). Hence the new $Q(B, s)$ in (6.2) has $N-1$ branching points and the property (3.30) is generalized to

$$
Q(B, s)=c_{+} Q\left(s+i \sigma_{+}\right)+c_{-} Q\left(s+i \sigma_{-}\right)+\sum_{j=4}^{N} c_{j} Q\left(s+i \sigma_{j}\right),
$$

where $\left\{-i \sigma_{+},-i \sigma_{-},-i \sigma_{j}\right\}(j=4, \cdots, N)$ are the branching points, i.e. the zeroes of the denominator of $Q(B, s)$. In this case one has to solve an algebraic equation of $(N-1)$ th order, whose zeroes do not have in general an analytical expression. We seek a perturbative solution in $B$. It is lengthy, but straightforward, to obtain up to second order in $B$

$$
\left\{\begin{array}{l}
\sigma_{ \pm}= \pm B-B^{2} \sum_{j=4}^{N} \frac{f_{j}}{2 \delta_{j}} \\
\sigma_{j}=\delta_{j}+B^{2} \frac{f_{j}}{\delta_{j}}
\end{array},\left\{\begin{array}{l}
c_{ \pm}=1 / 2 \mp B \sum_{j=4}^{N} \frac{f_{j}}{4 \delta_{j}}-B^{2} \sum_{j=4}^{N} \frac{f_{j}}{2 \delta_{j}^{2}} \\
c_{j}=B^{2} \frac{f_{j}}{\delta_{j}^{2}}
\end{array} .\right.\right.
$$

From the above equations we see that the presence of off-resonant levels modifies the energies $\sigma_{ \pm}= \pm B$ of the two dressed states by a shift of order $B^{2}$ and creates $N-3$ new dressed states with energies $\delta_{j} \simeq \Omega_{j}-\Omega_{3}$, whose contribution to the self-energy function is of order $B^{2}$.

By starting with the self-energy function (6.3) and looking for the location of the pole one obtains instead of Eq. (3.36) the following expression for the modified decay rate

$$
\begin{gathered}
\gamma_{\text {many }}(B)=\gamma\left[c_{-}\left(1-\frac{\sigma_{-}}{\omega_{0}}\right)^{\kappa}+c_{+}\left(1-\frac{\sigma_{+}}{\omega_{0}}\right)^{\kappa} \theta\left(\omega_{0}-\sigma_{+}\right)\right. \\
\left.+\sum_{\ell=4}^{N} c_{\ell}\left(1-\frac{\sigma_{\ell}}{\omega_{0}}\right)^{\kappa} \theta\left(\omega_{0}-\sigma_{\ell}\right)\right]
\end{gathered}
$$

where $\kappa=2 j \mp 1$.

By substituting the expressions (6.4) for the zeroes and the coefficients, valid up to second order in $B$, into Eq. (6.5) one gets

$$
\begin{aligned}
\gamma_{\text {many }}(B) & \simeq \gamma\left\{1+\kappa \frac{B^{2}}{\omega_{0}^{2}}+\frac{B^{2}}{\omega_{0}^{2}} \sum_{\ell=4}^{N} f_{\ell}\left[\left(\kappa \frac{\omega_{0}}{\delta_{\ell}}-\frac{\omega_{0}^{2}}{\delta_{\ell}^{2}}\right)-\left(\frac{\omega_{0}}{\delta_{\ell}}-\frac{\omega_{0}^{2}}{\delta_{\ell}^{2}}\right) \theta\left(\omega_{0}-\delta_{\ell}\right)\right]\right\} \\
& =\gamma(B)+\gamma \frac{B^{2}}{\omega_{0}^{2}} \sum_{\ell=4}^{N} f_{\ell}\left[\left(\kappa \frac{\omega_{0}}{\delta_{\ell}}-\frac{\omega_{0}^{2}}{\delta_{\ell}^{2}}\right)-\left(\frac{\omega_{0}}{\delta_{\ell}}-\frac{\omega_{0}^{2}}{\delta_{\ell}^{2}}\right) \theta\left(\omega_{0}-\delta_{\ell}\right)\right]+\mathrm{O}\left(B^{3}\right),
\end{aligned}
$$


where $\gamma(B)$ is the decay rate (3.36) evaluated in the three-level approximation.

The above general expression can be evaluated in practical cases of interest. For instance, by assuming that the off-resonant levels are well separated from the three main levels, that is by assuming $\delta_{\ell}=\Omega_{\ell}-\Omega_{3}>\omega_{0}$, all dressed states other than $| \pm\rangle$ do not enter in Eq. (6.5), because their energies are larger than the energy $\omega_{0}$ of level $|2\rangle$, and Eq. (6.6) reads

$$
\gamma_{\text {many }}(B) \simeq \gamma\left[1+\kappa \frac{B^{2}}{\omega_{0}^{2}}\left(1+\sum_{\ell=4}^{N} f_{\ell} \frac{\omega_{0}}{\delta_{\ell}}\right)\right] \simeq \gamma\left(B^{*}\right),
$$

where

$$
B^{*}=B\left[1+\sum_{\ell=4}^{N} f_{\ell} \frac{\omega_{0}}{2 \delta_{\ell}}\right] .
$$

This is the correction sought: the effect of sufficiently off-resonant levels, $\delta_{\ell}>\omega_{0}$, modifies the decay rate (3.36), calculated in the three-level approximation, simply by changing $B$ into $B^{*}$. Observe that $f_{\ell}$ is a rapidly decreasing function of $\ell$ (polynomial fall-off in atomic systems). Notice also that $B^{*}>B$, hence the presence of the other levels enhances the effect discussed in Section 3 .

\section{Concluding remarks}

We have studied the evolution of an unstable system under the action of an external (laser) field. The dynamical evolution of level \#2 (initial state) is modified by the laser field, tuned at the transition frequency 1-3. For physically sensible values of the parameters, the decay of level \#2 is faster when the laser is present. Equations (3.35)-(3.36) (valid to 4th order in the coupling constant) express the new lifetime as a function of the "natural" one and other parameters characterizing the physical system. The initial state decays to the laser-dressed states with different lifetimes. We have obtained Eq. (3.35) in 3 different ways, deriving the Fermi golden rule from the timedependent Schrödinger equation, by making use of Laplace transforms, as in Section 3.3, or starting from the dressed states, as in (5.16)-(5.17), or as a consequence of a normalization condition, as in (B.13). We also computed, in Section 6, the corrections due to off-resonant levels. We emphasize that, since we always work in the WeisskopfWigner approximation, the conceptual problems related to state preparation [22] and deviation from exponential behavior [20, 11, 18, 19] were not considered.

In which sense is the phenomenon discussed in this paper an "inverse" quantum Zeno effect? If the situation $B \gg \Lambda$ were experimentally attainable, then decay would be hindered and one could reasonably speak of a quantum Zeno effect provoked by the "continuous" observation performed on the system by the laser beam. On the other hand, when $B \ll \Lambda$, one can still think in terms of a "continuous gaze" of the laser on the system, but this enhances (rather than hinder) decay. One should also notice that the inclusion of the spontaneous decay of level \#3 in the Hamiltonian (2.7) would not change our conclusion (up to order $\Gamma_{13} / B$ ). The interpretation in 
terms of an "inverse" quantum Zeno effect is appealing and enables one to look at the problem from a different perspective.

Acknowledgments We thank E. Mihokova, G. Scamarcio and L.S. Schulman for useful discussions.

\section{Appendix A}

We discuss here some general properties of the matrix elements and derive Eqs. (3.15)(3.16) of the text. An exhaustive analysis of some general features of the matrix elements can be found in [29], but we will focus here on the behavior at small and large values of $\omega$. The matrix elements (2.2) of the 1-2 interaction Hamiltonian read

$$
\phi_{\boldsymbol{k} \lambda}=\sqrt{\frac{2 \pi \alpha}{V \omega}} \int d^{3} x e^{-i \boldsymbol{k} \cdot \boldsymbol{x}} \boldsymbol{\epsilon}_{\boldsymbol{k} \lambda}^{*} \cdot \boldsymbol{j}_{12}(\boldsymbol{x}),
$$

where $\alpha=e^{2} / 4 \pi \epsilon_{0}$ is the fine-structure constant. If the wavelength of the radiation is large compared to the size $a$ of the system (i.e. $\omega \ll \Lambda=a^{-1}$ ) the main contribution to the integral (A.1) comes from small values of $r=|\boldsymbol{x}|(\omega r \ll 1)$. Expanding the exponential $(\boldsymbol{k}=\boldsymbol{n} \omega)$

$$
\exp (-i \boldsymbol{k} \cdot \boldsymbol{x}) \equiv \exp (-i \omega \boldsymbol{n} \cdot \boldsymbol{x})=1-i \omega(\boldsymbol{n} \cdot \boldsymbol{x})+\frac{(-i \omega)^{2}}{2 !}(\boldsymbol{n} \cdot \boldsymbol{x})^{2}+\ldots
$$

and integrating term by term one obtains the asymptotic series

$$
\phi_{\boldsymbol{k} \lambda} \sim \sqrt{\frac{2 \pi}{V}} \sqrt{\frac{\alpha}{\omega}} \sum_{s=0}^{\infty} q_{\boldsymbol{n} \lambda}^{(s)} \omega^{s},
$$

where

$$
q_{\boldsymbol{n} \lambda}^{(s)} \equiv \frac{(-i)^{s}}{s !} \int d^{3} x \boldsymbol{\epsilon}_{\boldsymbol{n} \lambda}^{*} \cdot \boldsymbol{j}_{12}(\boldsymbol{x})(\boldsymbol{n} \cdot \boldsymbol{x})^{s}
$$

$\left(\boldsymbol{\epsilon}_{\boldsymbol{k} \lambda}=\boldsymbol{\epsilon}_{\boldsymbol{n} \lambda}\right.$ depends only on the direction of $\left.\boldsymbol{k}\right)$. Notice that we explicitly wrote every $\omega$-dependence and that $q^{(s)}$ does not depend on $\omega$. Observe that $q^{(0)}$ corresponds to electric-dipole transitions E1, $q^{(1)}$ to electric quadrupole E2 and/or magnetic dipole transitions M1, and so on. Hence $s=j-\lambda$, where $\lambda=0(\lambda=1)$ stands for magnetic (electric) transition $\mathrm{Mj}(\mathrm{Ej})$. Since the dominant contribution to the integral in (A.4) comes from a region of size $a$ and the current $j_{12}$ is essentially $\omega_{0} / a^{2}$, we get

$$
q_{\boldsymbol{n} \lambda}^{(s)} \propto \omega_{0} a^{s+1}, \quad \omega_{0} \equiv E_{2}-E_{1} .
$$

If $\omega a \ll 1$ the dominant term in the series (A.3) is the first nonvanishing one, namely

$$
\phi_{\boldsymbol{k} \lambda} \sim q_{\boldsymbol{n} \lambda}^{(r)} \omega^{r} \propto\left(\omega_{0} a\right)(\omega a)^{r}
$$


for some $s=r$. In the continuum limit one gets

$$
\begin{aligned}
\sum_{\boldsymbol{k}, \lambda}\left|\phi_{\boldsymbol{k} \lambda}\right|^{2} \longrightarrow \frac{V}{(2 \pi)^{3}} \sum_{\lambda} \int d^{3} k\left|\phi_{\boldsymbol{k} \lambda}\right|^{2} & =\int_{0}^{\infty} d \omega \omega^{2} \frac{V}{(2 \pi)^{3}} \sum_{\lambda} \int d \Omega\left|\phi_{\boldsymbol{k} \lambda}\right|^{2} \\
& =\int_{0}^{\infty} d \omega g^{2} \omega_{0} \chi^{2}(\omega),
\end{aligned}
$$

where we have defined

$$
g^{2} \omega_{0} \chi^{2}(\omega) \equiv \lim _{V \rightarrow \infty} \frac{\omega^{2} V}{(2 \pi)^{3}} \sum_{\lambda} \int d \Omega\left|\phi_{\boldsymbol{k} \lambda}\right|^{2}
$$

as in (3.14). From (A.6) we obtain

$$
\left|\phi_{\boldsymbol{k} \lambda}\right|^{2}=\frac{2 \pi}{V} \frac{\alpha}{\omega}\left|\sum_{r=0}^{\infty} q_{\boldsymbol{n} \lambda}^{(r)} \omega^{r}\right|^{2} \sim \frac{2 \pi}{V} \alpha\left|q_{\boldsymbol{n} \lambda}^{(r)}\right|^{2} \omega^{2 r-1}
$$

and therefore

$$
g^{2} \omega_{0} \chi^{2}(\omega) \sim \frac{\alpha}{(2 \pi)^{2}}\left(\sum_{\lambda} \int d \Omega\left|q_{\boldsymbol{n} \lambda}^{(r)}\right|^{2}\right) \omega^{2 r+1} \propto\left[\alpha\left(\omega_{0} a\right)^{2 r+2}\right] \omega_{0}\left(\frac{\omega}{\omega_{0}}\right)^{2 r+1} .
$$

Remembering that $2 r+1=2 j-2 \lambda+1=2 j \mp 1$, we obtain the first equation in (3.15) and Eq. (3.16).

On the other hand, if the wavelength is much smaller than $a$ (i.e. $\omega \gg \Lambda$ ), we first rewrite (A.1) in the following form

$$
\phi_{\boldsymbol{k} \lambda}=\sqrt{\frac{2 \pi \alpha}{V \omega}} \int d^{3} x e^{-i \omega \boldsymbol{n} \cdot \boldsymbol{x}} \boldsymbol{\epsilon}_{\boldsymbol{k} \lambda}^{*} \cdot \boldsymbol{j}_{12}(\boldsymbol{x})=\sqrt{\frac{2 \pi \alpha}{V \omega}} \int d x_{\|} e^{-i \omega x_{\|}} j_{\boldsymbol{n} \lambda, 12}\left(x_{\|}\right),
$$

where

$$
j_{\boldsymbol{n} \lambda, 12}\left(x_{\|}\right) \equiv \int d^{2} x_{\perp} \boldsymbol{\epsilon}_{\boldsymbol{n} \lambda}^{*} \cdot \boldsymbol{j}_{12}(\boldsymbol{x})
$$

and $\boldsymbol{x} \equiv x_{\|} \boldsymbol{n}+\boldsymbol{x}_{\perp}$. According to the Riemann-Lesbegue lemma, the integral in (A.11) vanishes in the $\omega \rightarrow \infty$ limit. In particular, if $j_{\boldsymbol{n} \lambda, 12}\left(x_{\|}\right)$is $N$ times differentiable, integrating by parts we get

$$
\phi_{\boldsymbol{k} \lambda}=\sqrt{\frac{2 \pi \alpha}{V \omega}} \frac{1}{(i \omega)^{N}} \int d x e^{-i \omega x} \frac{d^{N}}{d x^{N}} j_{\boldsymbol{n} \lambda, 12}(x)
$$

and we can write

$$
\phi_{\boldsymbol{k} \lambda}=o\left(\omega^{-N-1 / 2}\right), \quad(\omega \gg \Lambda)
$$

which yields the large $\omega$ behavior of the second equation in (3.15). It goes without saying that if $j_{\boldsymbol{n} \lambda, 12}\left(x_{\|}\right)$is an analytic function, then $\phi_{\boldsymbol{k} \lambda} \rightarrow 0$ more rapidly than any power. The second equation in (3.15) is therefore a conservative estimate. 


\section{Appendix B}

In this Appendix we shall analyze the spectrum of the emitted photons. We start by substituting (4.1) into (3.10) and (3.11), to obtain

$$
\begin{gathered}
\widetilde{y}_{\boldsymbol{k} \lambda}(s)=\frac{-i \phi_{\boldsymbol{k} \lambda}\left(s+i \omega_{k}\right)}{\left(s+i \omega_{k}\right)^{2}+B^{2}} \frac{1}{s-s_{\text {pole }}} \\
\widetilde{z}_{\boldsymbol{k} \lambda}(s)=-\frac{\sqrt{\bar{N}_{0}} \Phi_{\boldsymbol{k}_{0} \lambda_{0}}^{*} \phi_{\boldsymbol{k} \lambda}}{\left(s+i \omega_{k}\right)^{2}+B^{2}} \frac{1}{s-s_{\text {pole }}} .
\end{gathered}
$$

Closing the Bromwich path with a semicircle in the half plane Res $<0$, we get

$$
\begin{aligned}
y_{\boldsymbol{k} \lambda}(t) & =\frac{1}{2 \pi i} \int_{\Gamma} d s e^{t s} \widetilde{y}_{\boldsymbol{k} \lambda}(s), \\
z_{\boldsymbol{k} \lambda}(t) & =\frac{1}{2 \pi i} \int_{\Gamma} d s e^{t s} \widetilde{z}_{\boldsymbol{k} \lambda}(s),
\end{aligned}
$$

which can be evaluated by summing over the integrand residues. The quantity $\left|y_{\boldsymbol{k} \lambda}(t)\right|^{2}\left(\left|z_{\boldsymbol{k} \lambda}(t)\right|^{2}\right)$ represents the probability that, at time $t$, the transition $2 \rightarrow 1$ $(2 \rightarrow 1 \rightarrow 3)$ has taken place. When $t \rightarrow \infty$, the contribution of $s_{\text {pole }}$ (that has a finite negative real part) is exponentially damped. This leaves only the contributions of the poles in $-i\left(\omega_{k} \pm B\right)$.

We look first at the case $B=0$ (laser off). One gets $\left(z_{\boldsymbol{k} \lambda}=0, \forall t\right)$

$$
\left|y_{\boldsymbol{k} \lambda}(+\infty)\right|^{2}=\frac{\left|\phi_{\boldsymbol{k} \lambda}\right|^{2}}{\left(\omega_{k}-\bar{\omega}_{0}\right)^{2}+\gamma^{2} / 4}
$$

and, in the continuum limit (3.14), the probability to emit a photon in the frequency range $(\omega, \omega+d \omega)$ reads

$$
d P_{B=0}=g^{2} \omega_{0} \chi^{2}(\omega) f_{L}\left(\omega-\bar{\omega}_{0} ; \gamma\right) d \omega
$$

where $f_{L}$ is the Lorentzian profile

$$
f_{L}(\omega ; \gamma)=\frac{1}{\omega^{2}+\gamma^{2} / 4}
$$

This is Eq. (4.3) of the text. The quantity $P$ must be normalized to unity: imposing this condition one gets the Fermi golden rule (3.28).

On the other hand, when $B \neq 0$, the total emission probability is given by the sum

$$
\left|y_{\boldsymbol{k} \lambda}(\infty)\right|^{2}+\left|z_{\boldsymbol{k} \lambda}(\infty)\right|^{2}
$$

and it is straightforward to derive the following expressions $\left(\nu_{k}=\omega_{k}-\bar{\omega}_{0}\right.$ and we write for simplicity $\gamma(B)=\gamma)$

$\left|y_{\boldsymbol{k} \lambda}(\infty)\right|^{2}=\frac{\left|\phi_{\boldsymbol{k} \lambda}\right|^{2}}{\left|\left(\nu_{k}+i \frac{\gamma}{2}\right)^{2}-B^{2}\right|^{2}}\left[\left(\nu_{k}^{2}+\frac{\gamma^{2}}{4}\right) \cos ^{2}(B t)+B^{2} \sin ^{2}(B t)+\frac{\gamma B}{2} \sin (2 B t)\right]$, 


$$
\left|z_{\boldsymbol{k} \lambda}(\infty)\right|^{2}=\frac{\left|\phi_{\boldsymbol{k} \lambda}\right|^{2}}{\left|\left(\nu_{k}+i \frac{\gamma}{2}\right)^{2}-B^{2}\right|^{2}}\left[\left(\nu_{k}^{2}+\frac{\gamma^{2}}{4}\right) \sin ^{2}(B t)+B^{2} \cos ^{2}(B t)-\frac{\gamma B}{2} \sin (2 B t)\right]
$$

which yield

$$
\left|y_{\boldsymbol{k} \lambda}(\infty)\right|^{2}+\left|z_{\boldsymbol{k} \lambda}(\infty)\right|^{2}=\frac{\left|\phi_{\boldsymbol{k} \lambda}\right|^{2}}{\left|\left(\nu_{k}+i \frac{\gamma}{2}\right)^{2}-B^{2}\right|^{2}}\left(\nu_{k}^{2}+\frac{\gamma^{2}}{4}+B^{2}\right)
$$

Therefore, in the continuum limit, we can write

$$
d P_{B}=g^{2} \omega_{0} \chi^{2}(\omega) \frac{\left(\omega-\bar{\omega}_{0}\right)^{2}+\frac{\gamma^{2}}{4}+B^{2}}{\left[\left(\omega-\bar{\omega}_{0}-B\right)^{2}+\frac{\gamma^{2}}{4}\right]\left[\left(\omega-\bar{\omega}_{0}+B\right)^{2}+\frac{\gamma^{2}}{4}\right]} d \omega .
$$

This formula can be rewritten in the following form

$$
d P_{B}=g^{2} \omega_{0} \chi^{2}(\omega) \frac{1}{2}\left[f_{L}\left(\omega-\bar{\omega}_{0}-B ; \gamma\right)+f_{L}\left(\omega-\bar{\omega}_{0}+B ; \gamma\right)\right] d \omega
$$

This is Eq. (4.5) of the text. We see that the emission probability is the sum of two Lorentzians, centered in $\bar{\omega}_{0}-B$ and $\bar{\omega}_{0}+B$ and weighted by $g^{2} \omega_{0} \chi^{2}(\omega)$. This result is in agreement with that obtained in Refs. [16, 32]. Incidentally, we notice that the value (3.34) of $\gamma(B)$ can be readily estimated by imposing the normalization of the emission probability

$$
\int d P_{B}=\int_{0}^{\infty} g^{2} \omega_{0} \chi^{2}(\omega) \frac{1}{2}\left[f_{L}\left(\omega-\bar{\omega}_{0}-B ; \gamma\right)+f_{L}\left(\omega-\bar{\omega}_{0}+B ; \gamma\right)\right] d \omega=1
$$

Performing the integration one obtains $\left(\gamma \ll \bar{\omega}_{0}\right.$, hence one can integrate over the whole real axis and take $\chi^{2}(\omega)$ equal to its value on each Lorentzian peak)

$$
1=\int d P_{B} \approx \frac{1}{2} g^{2} \omega_{0}\left[\chi^{2}\left(\bar{\omega}_{0}+B\right)+\chi^{2}\left(\bar{\omega}_{0}-B\right)\right] \frac{2 \pi}{\gamma(B)}
$$

which yields Eq. (3.34) of the text.

\section{References}

[1] A. Beskow and J. Nilsson, Arkiv für Fysik 34, 561 (1967); L.A. Khalfin, Zh. Eksp. Teor. Fiz. Pis. Red. 8, 106 (1968) [JETP Letters 8, 65 (1968)]; L. Fonda, G.C. Ghirardi, A. Rimini and T. Weber, Nuovo Cim. A15, 689 (1973); A18, 805 (1973).

[2] B. Misra and E.C.G. Sudarshan, J. Math. Phys. 18, 756 (1977).

[3] E. Mihokova, S. Pascazio and L.S. Schulman, Phys. Rev. A56, 25 (1997). 
[4] L.S. Schulman, Phys. Rev. A57, 1509 (1998).

[5] R.J. Cook, Phys. Scr. T21, 49 (1988).

[6] W.H. Itano, D.J. Heinzen, J.J. Bollinger and D.J. Wineland, Phys. Rev. A41, 2295 (1990). This experiment gave rise to a lively discussion. See: A. Beige and G. Hegerfeldt, Phys. Rev. A53, 53 (1996) and references therein.

[7] S. Pascazio, M. Namiki, G. Badurek and H. Rauch, Phys. Lett. A179, 155 (1993); S. Pascazio and M. Namiki, Phys. Rev. A50, 4582 (1994).

[8] P. Kwiat, H. Weinfurter, T. Herzog, A. Zeilinger and M. Kasevich, Phys. Rev. Lett. 74, 4763 (1995).

[9] A Luis and J. Peřina, Phys. Rev. Lett. 76, 4340 (1996).

[10] G. Gamow, Z. Phys. 51, 204 (1928); V. Weisskopf and E.P. Wigner, Z. Phys. 63, 54 (1930); 65, 18 (1930); G. Breit and E.P. Wigner, Phys. Rev. 49, 519 (1936).

[11] E. Fermi, Rev. Mod. Phys. 4, 87 (1932); Nuclear Physics (Univ. Chicago, Chicago, 1950) pp. 136, 148; See also Notes on Quantum Mechanics. A Course Given at the University of Chicago in 1954, edited by E Segré (Univ. Chicago, Chicago, 1960) Lec. 23.

[12] S. Pascazio and P. Facchi, Acta Physica Slovaca 49, 557 (1999) quantph/9904076).

[13] A.G. Kofman and G. Kurizki, Acta Physica Slovaca 49, 541 (1999). See also A.G. Kofman and G. Kurizki, Phys. Rev. A54, R3750 (1996); G. Harel, A.G. Kofman A. Kozhekin and G. Kurizki, Optics Express 2, 355 (1998).

[14] M.B. Plenio, P.L. Knight and R.C. Thompson, Opt. Comm. 123, 278 (1996).

[15] K. Kraus, Found. Phys. 11, 547 (1981).

[16] S.-Y. Zhu, L.M. Narducci and M.O. Scully, Phys. Rev. A52, 4791 (1995). See also S.-Y. Zhu and M.O. Scully, Phys. Rev. Lett 76, 388 (1996); H. Huang, S.-Y. Zhu M.S. Zubairy and M.O. Scully,, Phys. Rev. A53, 1834 (1996).

[17] S.R. Wilkinson et al., Nature 387, 575 (1997).

[18] M. Hillery, Phys. Rev. A24, 933 (1981).

[19] L. Mandelstam and I. Tamm, J. Phys. 9, 249 (1945); V. Fock and N. Krylov, J. Phys. 11, 112 (1947); E.J. Hellund, Phys. Rev. 89, 919 (1953); M. Namiki and N. Mugibayashi, Prog. Theor. Phys. 10, 474 (1953). L.A. Khalfin, Dokl. Acad. Nauk USSR 115, 277 (1957) [Sov. Phys. Dokl. 2, 340 (1957)]; Zh. Eksp. Teor. Fiz. 33, 1371 (1958)[Sov. Phys. JET 6, 1053 (1958)]. 
[20] H. Nakazato, M. Namiki and S. Pascazio, Int. J. Mod. Phys. B10, 247 (1996).

[21] C. Bernardini, L. Maiani and M. Testa, Phys. Rev. Lett. 71, 2687 (1993); L. Maiani and M. Testa, Ann. Phys. (NY) 263, 353 (1998).

[22] P. Facchi and S. Pascazio, Phys. Lett. A241, 139 (1998).

[23] I. Joichi, Sh. Matsumoto and M. Yoshimura, Phys. Rev. D58, 043507; 045004 (1998).

[24] R.F. Alvarez-Estrada and J.L. Sánchez-Gómez, Phys. Lett. A253, 252 (1999).

[25] B. R. Mollow, Phys. Rev. A12, 1919 (1975); C. Cohen-Tannoudji, J. DupontRoc and G. Grynberg, Atom-Photon Interactions: Basic Processes and Applications (Wiley, New York 1998).

[26] K.J. Boller, A. Imamoglu and S.E. Harris, Phys. Rev. Lett. 66, 2593 (1991); J.E. Field, K.H. Hahn and S.E. Harris, Phys. Rev. Lett. 67, 3062 (1991); S.P. Tewari and G.S.Agarwal, Phys. Rev. Lett. 56, 1811 (1986); S.E. Harris, J.E. Field and A. Imamoglu, Phys. Rev. Lett. 64, 1107 (1990).

[27] I. Tamm, J. Phys. (USSR) 9, 449 (1945); S. Dancoff, Phys. Rev. 78, 382 (1950).

[28] P.M. Radmore and P.L. Knight, J. Phys. (At. Mol. Phys.) B15, 561 (1982); P.L. Knight and M.A. Lauder, Phys. Rep. 190, 1 (1990).

[29] V.B. Berestetskii, E.M. Lifshits and L.P. Pitaevskii, Quantum electrodynamics, Course of Theor. Phys., Vol. 4 (Pergamon Press, Oxford, 1982), Chapter 5; H.E. Moses, Lett. Nuovo Cimento 4 51; 54 (1972); Phys. Rev. A8 1710 (1973); J. Seke, Physica A203 269; 284 (1994).

[30] U. Fano, Phys. Rev. 124, 1866 (1961); C. Cohen-Tannoudji and S. Reynaud, J. Phys. B10, 345; 365; 2311 (1977); H.-I. Yoo and J.H. Eberly, Phys. Rep. 118, 239 (1985).

[31] S. H. Autler and C. H. Townes, Phys. Rev. 100, 703 (1955); C. H. Townes and A. L. Schawlow, Microwave Spectroscopy (Dover, New York, 1975).

[32] L.S. Schulman, J. Phys. A30, L293 (1997). 